Eugenio Montefusco $\cdot$ Benedetta Pellacci · Marco Squassina

\title{
Semiclassical states for weakly coupled nonlinear Schrödinger systems
}

Received April 12, 2006 and in revised form May 22, 2006

\begin{abstract}
We consider systems of weakly coupled Schrödinger equations with nonconstant potentials and investigate the existence of nontrivial nonnegative solutions which concentrate around local minima of the potentials. We obtain sufficient and necessary conditions for a sequence of least energy solutions to concentrate.
\end{abstract}

Keywords. Weakly coupled nonlinear Schrödinger systems, concentration phenomena, semiclassical limit, ground states, critical point theory, Clarke's subdifferential

\section{Introduction}

Starting from the celebrated works [8, 12, 26], the recent years have been marked out by an ever-growing interest in the study of standing wave solutions to the semilinear Schrödinger equation (NLS)

$$
i \phi_{t}+\Delta \phi+|\phi|^{2} \phi=0 \quad \text { in } \mathbb{R}^{3} \times(0, \infty),
$$

where $i$ denotes the imaginary unit. As a related problem, a large amount of work (see [1, 4, 5, 13, 15, 20, 27] and references therein) has been devoted to the study of the semiclassical states for (NLS), namely the study of the singularly perturbed equation $-\varepsilon^{2} \Delta u+V(x) u=u^{3}$ in $\mathbb{R}^{3}$ for $\varepsilon$ going to zero, where $V(x)$ is a potential modeling the action of external forces. Under different hypotheses on the potential $V$ it has been proved that there exists a family $\left\{u_{\varepsilon}\right\}$ of solutions which exhibits a spike shape around the nondegenerate critical points of $V$ and decays elsewhere.

From a physical point of view, the nonlinear Schrödinger equation arises in the study of nonlinear optics in isotropic materials, for instance the propagation of pulses in a single-mode nonlinear optical fiber. However, a single-mode optical fiber is actually $b i$ modal due to the presence of some birefringence effects which tend to split a pulse into

E. Montefusco: Dipartimento di Matematica, Università degli Studi di Roma "La Sapienza", P.le A. Moro 5, I-00185 Roma, Italy; e-mail: montefusco@mat.uniroma1.it

B. Pellacci: Dipartimento di Scienze Applicate, Università degli Studi di Napoli "Parthenope", Via De Gasperi 5, I-80133 Napoli, Italy; e-mail: benedetta.pellacci@uniparthenope.it

M. Squassina: Dipartimento di Informatica, Università degli Studi di Verona, Cà Vignal 2, Strada Le Grazie 15, I-37134 Verona, Italy; e-mail: marco.squassina@univr.it

Mathematics Subject Classification (2000): 34B18, 34G20, 35Q55 
two pulses in two different polarization directions. Menyuk [19] showed that, under various simplifications and variable scalings, the complex amplitudes of the two wave packets $\phi$ and $\psi$ in a birefringence optical fiber are governed by a system of two coupled nonlinear Schrödinger equations ((CNLS) for short). Looking for standing wave solutions leads to study the following elliptic system:

$$
\begin{cases}-\Delta u+u=u^{3}+b v^{2} u & \text { in } \mathbb{R}^{3} \\ -\Delta v+\omega^{2} v=v^{3}+b u^{2} v & \text { in } \mathbb{R}^{3}\end{cases}
$$

where $b$ is a real-valued cross phase coefficient depending upon the anisotropy of the fiber, and $\omega$ is the frequencies ratio of the two waves. Physically, $b>0$ is known as the attractive case, whereas $b<0$ is the repulsive case. Apart from some special cases, the study of (1.1) is pretty complicated. This is because of the presence of semitrivial or scalar solutions: indeed, there always exist the solutions $(u, 0),(0, v)$ with $u, v$ solutions of the single equations in (1.1); then it becomes physically relevant to know whether or not a solution found is really vectorial, i.e. with both components nontrivial. Recently, this problem has been tackled in [2, 3, 7, 17, 18, 25] by different methods. In particular, in [18] it has been proved that for $b$ sufficiently small every ground state solution necessarily has one trivial component, while for $b$ sufficiently large the ground state solutions have both components positive. As far as the semiclassical states are concerned, we are naturally led to study the system

$$
\begin{cases}-\varepsilon^{2} \Delta u+V(x) u=u^{3}+b v^{2} u & \text { in } \mathbb{R}^{3} \\ -\varepsilon^{2} \Delta v+W(x) v=v^{3}+b u^{2} v & \text { in } \mathbb{R}^{3}\end{cases}
$$

This is the goal of this paper. We will assume that the potentials $V, W$ are Hölder continuous functions in $\mathbb{R}^{3}$, bounded from below away from zero, and $\varepsilon$ is a small parameter which will approach zero. Our intent is to show the existence, for small $\varepsilon$, of a nonnegative (i.e. with nonnegative components) least energy solution $\left(u_{\varepsilon}, v_{\varepsilon}\right)$ and then to prove sufficient and necessary conditions concerned with the concentration of $\left(u_{\varepsilon}, v_{\varepsilon}\right)$ around the local minimum (possibly degenerate) points of the potentials, which are supposed to be in the same region. Aiming to use variational methods, we will consider the functional $J_{\varepsilon}$ associated to $\sqrt{S_{\varepsilon}}$, which satisfies all the assumptions of the mountain pass theorem ([6]) except for the Palais-Smale condition since we do not assume any global condition on $V, W$. Then we will use a vectorial adaptation of the argument in [13]; namely, we will perform a penalization of $J_{\varepsilon}$, exploiting the homogeneity of the nonlinearities, outside the region containing the minimum points of the potentials, so that we will consider a modified functional which satisfies all the hypotheses of the mountain pass theorem including the Palais-Smale condition. To show the concentration, we will argue on the sum $u_{\varepsilon}(x)+v_{\varepsilon}(x)$ proving that it is uniformly, with respect to $\varepsilon$, bounded away from zero, and by taking advantage of the known properties of the autonomous system we can show that $u_{\varepsilon}(x)+v_{\varepsilon}(x)$ has exactly one global maximum point, which tends to a minimum point of $V$ or $W$. Here we cannot be more precise without assuming some conditions on $b$, as one of $u_{\varepsilon}$ and $v_{\varepsilon}$ may vanish as $\varepsilon \rightarrow 0$. Namely, we can show that for $b$ smaller than 
a positive constant $b_{0}$ (defined in (2.5)) either $u_{\varepsilon}$ or $v_{\varepsilon}$ necessarily expires and the other tends - up to scalings - to the least energy solution of the corresponding autonomous nonlinear Schrödinger equation. When $b$ is large (greater than a positive constant $b_{1}$ defined in (2.5) both $u_{\varepsilon}$ and $v_{\varepsilon}$ survive and we recover a least energy vectorial solution of the autonomous system (see Theorem 2.1). As physically reasonable, for materials with low anisotropy, one component of the system dominates the other, since the low birefringence is not able to split a soliton-type solution in two distinct pulses. Recently, the repulsive case $b<0$ (a model for the Bose-Einstein condensation) was studied in [21]. We stress that the methods used therein are very different from ours, since the change of sign of the constant $b$ produces a different behavior of the solutions (see also [14] for the case of a single equation).

Concerning the necessary conditions for a sequence of solutions to concentrate, in contrast to the scalar case with power nonlinearity $([1,5])$, we cannot in general derive an explicit representation of the so called ground energy function $\Sigma$ (see formulas 2.8 ) (2.9)). The underlying philosophy is that when the limit problem (1.1) lacks uniqueness, the ground energy function, which will be shown to be at least locally Lipschitz continuous, may lose its additional smoothness properties. Nevertheless, in this framework, along the lines of [24], we prove that a necessary condition for a family of solutions $\left(u_{\varepsilon}, v_{\varepsilon}\right)$ to concentrate around a given point $z$ is that $z$ is a critical point, not necessarily a minimum point, of $\Sigma$ in the sense of the Clarke subdifferential $\partial_{C}$, that is, $0 \in \partial_{C} \Sigma(z)$. Moreover, due to the previously mentioned characterization of least energy solutions in terms of the coupling parameter $b$ (see Proposition 3.18), we partition the concentration points $\mathcal{E}$ into three classes $\mathcal{E}=\mathcal{E}_{V} \cup \mathcal{E}_{W} \cup \mathcal{E}_{\Sigma}$, where

$$
\mathcal{E}_{V} \times \mathcal{E}_{W} \times \mathcal{E}_{\Sigma} \subset \operatorname{Crit}(V) \times \operatorname{Crit}(W) \times \operatorname{Crit}_{C}(\Sigma) .
$$

Here $\operatorname{Crit}(f)$ (resp. Crit $_{C}(f)$ ) denotes the set of classical critical points (resp. critical points in the sense of the Clarke subdifferential) of a function $f$. In this partition we can see again that if a family of solutions concentrates around a given point then we derive as a limit problem either a single equation or the entire system, depending on the value of $b$. Namely, we will find some positive constants $b_{0}^{\infty}<b_{1}^{\infty}<b_{2}^{\infty}$ such that for $b<b_{0}^{\infty}$ we obtain a single equation as the limit problem, for $b>b_{2}^{\infty}$ we show that if a family of least energy solutions concentrates, then its scaling around a minimum point of the potentials converges to a real vectorial least energy solution of the autonomous system.

The plan of the paper is the following. In Section 2 we introduce the functional setting and the statements of the main results. In Section 3 we prove sufficient conditions for concentration, and in Section 4 necessary conditions.

\section{The functional framework and main statements}

Let $V(x)$ and $W(x)$ be Hölder continuous functions in $\mathbb{R}^{3}$ and suppose that there exists a positive constant $\alpha$ such that

$$
V(x), W(x) \geq \alpha \quad \text { for all } x \in \mathbb{R}^{3} .
$$


In order to study $\left(S_{\varepsilon}\right)$ we use variational methods, so that we introduce the Hilbert space

$$
\mathbb{H}=\left\{(u, v) \in H^{1} \times H^{1}: \int_{\mathbb{R}^{3}} V(x) u^{2}<\infty, \quad \int_{\mathbb{R}^{3}} W(x) v^{2}<\infty\right\},
$$

where $H^{1}=H^{1}\left(\mathbb{R}^{3}\right)$ is the usual first order Sobolev space in $\mathbb{R}^{3}$. The norm in $\mathbb{H}$ is $\|(u, v)\|_{\mathbb{H}}^{2}=\|u\|_{\varepsilon, V}^{2}+\|v\|_{\varepsilon, W}^{2}$, where

$$
\|u\|_{\varepsilon, V}^{2}=\varepsilon^{2}\|\nabla u\|_{2}^{2}+\int_{\mathbb{R}^{3}} V(x) u^{2}, \quad\|v\|_{\varepsilon, W}^{2}=\varepsilon^{2}\|\nabla v\|_{2}^{2}+\int_{\mathbb{R}^{3}} W(x) v^{2},
$$

$\varepsilon$ being a small parameter and $\|\cdot\|_{p}$ the standard norm in $L^{p}=L^{p}\left(\mathbb{R}^{3}\right)$ for $1 \leq p \leq \infty$. We will study the functional $J_{\varepsilon}: \mathbb{H} \rightarrow \mathbb{R}$ defined by

$$
J_{\varepsilon}(u, v)=\frac{1}{2}\|u\|_{\varepsilon, V}^{2}+\frac{1}{2}\|v\|_{\varepsilon, W}^{2}-\int_{\mathbb{R}^{3}} F(u, v),
$$

where we have set

$$
F(u, v)=\frac{1}{4}\left(u^{4}+2 b u^{2} v^{2}+v^{4}\right) \quad \text { with } b>0 .
$$

It is easily checked that $J_{\varepsilon}$ is well defined and of class $C^{1}$ on $\mathbb{H}$. A nontrivial solution of problem $\left(S_{\varepsilon}\right)$ is a couple $\left(u_{\varepsilon}, v_{\varepsilon}\right) \neq(0,0)$ in $\mathbb{H}$ which is a critical point of $J_{\varepsilon}$.

We denote by $B(x, r)$ the open ball centered at $x$ with radius $r$, and by $\partial B(x, r)$ its boundary.

Concerning sufficient conditions for concentration to occur, we will prove two main results; the first is the following.

Theorem 2.1. Assume 2.1 and that there exist $z \in \mathbb{R}^{3}$ and $r>0$ such that

$$
\begin{aligned}
V_{0} & =\min _{B(z, r)} V<\min _{\partial B(z, r)} V, \\
W_{0} & =\min _{B(z, r)} W<\min _{\partial B(z, r)} W .
\end{aligned}
$$

Then there exists $\varepsilon_{0}>0$ such that, for every $0<\varepsilon<\varepsilon_{0}$, problem $\left(S_{\varepsilon}\right)$ admits a nontrivial solution $\left(u_{\varepsilon}, v_{\varepsilon}\right) \in \mathbb{H}$, with $u_{\varepsilon}, v_{\varepsilon} \geq 0$, such that the following facts hold:

(i) $u_{\varepsilon}+v_{\varepsilon}$ has exactly one global maximum point $x_{\varepsilon} \in B(z, r)$ with

$$
\lim _{\varepsilon \rightarrow 0} V\left(x_{\varepsilon}\right)=V_{0} \quad \text { or } \quad \lim _{\varepsilon \rightarrow 0} W\left(x_{\varepsilon}\right)=W_{0} .
$$

Furthermore, there exist $\mu_{1}, \mu_{2}>0$ such that, for every $x \in \mathbb{R}^{3}$,

$$
u_{\varepsilon}(x)+v_{\varepsilon}(x) \leq \mu_{1} e^{-\mu_{2}\left|x-x_{\varepsilon}\right| / \varepsilon} .
$$


(ii) Define $b_{0}<b_{1}$ by

$$
b_{0}=\max \left\{\sqrt[4]{W_{0} / V_{0}}, \sqrt[4]{V_{0} / W_{0}}\right\}, \quad b_{1}=\max \left\{h\left(\sqrt{W_{0} / V_{0}}\right), h\left(\sqrt{V_{0} / W_{0}}\right)\right\},
$$

with

$$
h(s)=\min \left\{\frac{s}{32}\left(7+\frac{1}{s^{2}}\right)^{2}-1, \frac{s^{2}+3}{4}\right\} .
$$

Then the following facts hold:

- if $b<b_{0}$, then there exists $\sigma>0$ such that for all $0<\varepsilon<\varepsilon_{0}$,

$$
\text { either } u_{\varepsilon}\left(x_{\varepsilon}\right) \rightarrow 0 \text { and } v_{\varepsilon}\left(x_{\varepsilon}\right) \geq \sigma \text {, or } v_{\varepsilon}\left(x_{\varepsilon}\right) \rightarrow 0 \text { and } u_{\varepsilon}\left(x_{\varepsilon}\right) \geq \sigma \text {. }
$$

- if $b>b_{1}$, then there exist $\sigma>0$ such that, for all $0<\varepsilon<\varepsilon_{0}$,

$$
u_{\varepsilon}\left(x_{\varepsilon}\right) \geq \sigma, \quad v_{\varepsilon}\left(x_{\varepsilon}\right) \geq \sigma .
$$

Remarks 2.2. 1. Actually, we can be more precise in conclusion (ii) of Theorem 2.1 Indeed, if $V_{0}<W_{0}$ then $u_{\varepsilon}$ converges to zero while $v_{\varepsilon}\left(x_{\varepsilon}\right)$ remains bounded away from zero; otherwise if $W_{0}<V_{0}$ then $u_{\varepsilon}$ survives and $v_{\varepsilon}$ expires (see Remark 3.4 for more details).

2. In the case $V=W$, we have $b_{0}=b_{1}=1$. For $b<1,\left(u_{\varepsilon}, v_{\varepsilon}\right)$ converges (up to scalings) to the least energy solution of one of the equations in $\left(S_{\varepsilon}\right)$, while for $b>1$, $\left(u_{\varepsilon}, v_{\varepsilon}\right)$ converges to a real vector solution of the system $\left(S_{\varepsilon}\right)$.

3. The constants $b_{0}$ and $b_{1}$ depend only on the minima $V_{0}, W_{0}$, so that $V$ and $W$ may have a degenerate minimum point or a closed, connected bounded set of nonnegative measure of minimum points.

4. When considering the action of external forces in the propagation of pulses in optical fibers, the potentials in the model problem are $V(x)$ and $W(x)=V(x)+c$ with $c$ a positive constant. In this case the result follows just by assuming that 2.2$]$ holds.

We can also prove a more general result than Theorem 2.1 In order to do this, let us define the functional $I_{z}: H^{1} \times H^{1} \rightarrow \mathbb{R}$ with frozen potentials,

$$
I_{z}(u, v)=\frac{1}{2}\|u\|_{z}^{2}+\frac{1}{2}\|v\|_{z}^{2}-\int_{\mathbb{R}^{3}} F(u, v),
$$

where $\|u\|_{z}^{2}=\|\nabla u\|_{2}^{2}+V(z)\|u\|_{2}^{2}$ for every $u \in H^{1}$. The critical points of $I_{z}$ are the solutions of the system

$$
\begin{cases}-\Delta u+V(z) u=u^{3}+b v^{2} u & \text { in } \mathbb{R}^{3}, \\ -\Delta v+W(z) v=v^{3}+b u^{2} v & \text { in } \mathbb{R}^{3} .\end{cases}
$$

The Nehari manifold associated to $I_{z}$ is defined by

$$
\mathcal{N}_{z}=\left\{(u, v) \in H^{1} \times H^{1} \backslash\{(0,0)\}:\left\langle I_{z}^{\prime}(u, v),(u, v)\right\rangle=0\right\},
$$


and the infimum of $I_{z}$ on $\mathcal{N}_{z}$ is denoted by

$$
\Sigma(z)=\inf _{\mathcal{N}_{z}} I_{z}
$$

Following the same argument of Lemma 3.1 in [18], it is possible to prove that the mountain pass level of $I_{z}$ is equal to $\Sigma(z)$. In the following we let $\left(\varphi_{z}, \psi_{z}\right) \neq(0,0)$ denote the point where $I_{z}$ achieves $\Sigma(z)$, that is, $\left(\varphi_{z}, \psi_{z}\right)$ will be a least energy solution of $\left(S_{z}\right)$ (see [9] or [18], for example).

Because of this property, the function $\Sigma$ is known as the ground energy function and plays an important role when studying necessary and sufficient conditions for the concentration to occur, as the following result shows.

Theorem 2.3. Assume 2.1 and that there exist $z \in \mathbb{R}^{3}$ and $r>0$ such that

$$
\Sigma_{0}=\min _{B(z, r)} \Sigma<\min _{\partial B(z, r)} \Sigma
$$

Then there exists $\varepsilon_{0}>0$ such that, for every $0<\varepsilon<\varepsilon_{0}$, problem $\left(S_{\varepsilon}\right)$ admits a nontrivial solution $\left(u_{\varepsilon}, v_{\varepsilon}\right) \in \mathbb{H}$, with $u_{\varepsilon}, v_{\varepsilon} \geq 0$, such that $u_{\varepsilon}+v_{\varepsilon}$ has exactly one global maximum point $x_{\varepsilon} \in B(z, r)$ with

$$
\lim _{\varepsilon \rightarrow 0} \Sigma\left(x_{\varepsilon}\right)=\Sigma_{0}
$$

and conclusions (i) and (ii) of Theorem 2.1 hold true.

Remark 2.4. Theorem 2.3 is more general than Theorem 2.1. Indeed, conditions (2.2) (2.3) imply the desired information (2.10) (see for the details the proof in Section 2.1). However, Theorem 2.3 is an abstract result since we cannot write down explicitly the function $\Sigma$, due to the possible lack of uniqueness of least energy solutions of $\left(S_{z}\right)$. It would be interesting to see if, by assuming that $\Sigma$ has a 'topologically nontrivial' Clarke critical point, the concentration still pops up.

Aiming to state a necessary condition for a family of solutions $\left(u_{\varepsilon}, v_{\varepsilon}\right)$ to concentrate around a point $z$, we need a few preliminary definitions.

Definition 2.5. Let $z \in \mathbb{R}^{3}$ and let $b_{z} \geq 1$ be defined by

$$
b_{z}=\max \left\{\sqrt[4]{\frac{W(z)}{V(z)}}, \sqrt[4]{\frac{V(z)}{W(z)}}\right\} .
$$

For every $b>0$, we put

$$
\mathcal{O}_{b}=\left\{z \in \mathbb{R}^{3}: b_{z} \geq b\right\}
$$

Next we define the concentration sets. 
Definition 2.6. The concentration set for system $\left[S_{\varepsilon}\right]$ is defined by

$\mathcal{E}=\left\{z \in \mathbb{R}^{3}\right.$ : there exists a sequence of solutions $\left(u_{\varepsilon}, v_{\varepsilon}\right) \in \mathbb{H}$ of $\left(S_{\varepsilon}\right]$ with

$$
\begin{aligned}
& u_{\varepsilon}(z+\varepsilon x)+v_{\varepsilon}(z+\varepsilon x) \rightarrow 0 \text { as }|x| \rightarrow \infty \text { uniformly with respect } \\
& \text { to } \left.\varepsilon \text { and } \varepsilon^{-3} J_{\varepsilon}\left(u_{\varepsilon}, v_{\varepsilon}\right) \rightarrow \Sigma(z) \text { as } \varepsilon \rightarrow 0\right\} .
\end{aligned}
$$

We also introduce the following subsets of $\mathcal{E}$ :

$$
\begin{aligned}
& \mathcal{E}_{V}:=\left\{z \in \mathcal{E} \cap \mathcal{O}_{b}: u_{\mathcal{E}}(z) \geq \delta \text { for some } \delta>0 \text { and any } \varepsilon>0\right\}, \\
& \mathcal{E}_{W}:=\left\{z \in \mathcal{E} \cap \mathcal{O}_{b}: v_{\varepsilon}(z) \geq \delta \text { for some } \delta>0 \text { and any } \varepsilon>0\right\}, \\
& \mathcal{E}_{\Sigma}:=\mathcal{E} \backslash \mathcal{O}_{b} .
\end{aligned}
$$

In general the function $\Sigma$ is not known to be differentiable, but it is always locally Lipschitz, as we will see. On the other hand, we need to consider the critical points of $\Sigma$, so that we will use the Clarke subdifferential (see [11]), which is well defined for a locally Lipschitz function. We will need the following definition.

Definition 2.7. For $V, W \in C^{1}\left(\mathbb{R}^{3}\right)$ and $\Sigma \in \operatorname{Lip}_{\text {loc }}\left(\mathbb{R}^{3}\right)$ we denote by $\operatorname{Crit}(V)$ and $\mathrm{Crit}(W)$ the sets of critical points in $\mathcal{O}_{b}$ of $V$ and $W$ respectively, and by $\operatorname{Crit}_{C}(\Sigma)$ the set of critical points $z \notin \mathcal{O}_{b}$ of $\Sigma$ in the sense of Clarke subdifferential, that is,

$$
\begin{aligned}
\operatorname{Crit}(V) & =\left\{z \in \mathcal{O}_{b}: \nabla V(z)=0\right\}, \\
\operatorname{Crit}(W) & =\left\{z \in \mathcal{O}_{b}: \nabla W(z)=0\right\}, \\
\operatorname{Crit}_{C}(\Sigma) & =\left\{z \notin \mathcal{O}_{b}: \partial_{C} \Sigma(z) \ni 0\right\},
\end{aligned}
$$

where

$$
\partial_{C} \Sigma(z)=\left\{\eta \in \mathbb{R}^{3}: \Sigma^{0}(z ; w) \geq \eta \cdot w \text { for every } w \in \mathbb{R}^{3}\right\},
$$

$\Sigma^{0}(z ; w)$ being the generalized derivative of $\Sigma$ at $z$ along $w \in \mathbb{R}^{3}$, defined by

$$
\Sigma^{0}(z ; w)=\limsup _{\substack{\xi \rightarrow z \\ \lambda \rightarrow 0^{+}}} \frac{\Sigma(\xi+\lambda w)-\Sigma(\xi)}{\lambda} .
$$

We can now state the following necessary condition.

Theorem 2.8. Assume (2.1) and that $V, W \in C^{1}\left(\mathbb{R}^{3}\right)$ with

$$
|\nabla V(x)| \leq \beta e^{\gamma|x|} \text { and }|\nabla W(x)| \leq \beta e^{\gamma|x|},
$$

for all $x \in \mathbb{R}^{3}$ and for some constants $\beta>0$ and $\gamma \geq 0$. Then $\Sigma$ is locally Lipschitz continuous and the following facts hold:

(a) $\mathcal{E}_{V} \cap \mathcal{E}_{W} \cap\left\{z \in \mathbb{R}^{3}: V(z) \neq W(z)\right\}=\emptyset$ and

$$
\mathcal{E}=\mathcal{E}_{V} \cup \mathcal{E}_{W} \cup \mathcal{E}_{\Sigma}
$$

where

$$
\mathcal{E}_{V} \times \mathcal{E}_{W} \times \mathcal{E}_{\Sigma} \subset \operatorname{Crit}(V) \times \operatorname{Crit}(W) \times \operatorname{Crit}_{C}(\Sigma)
$$


(b) If $V, W \in L^{\infty}$, let $b_{0}^{\infty}<b_{1}^{\infty}<b_{2}^{\infty}$ be defined by

$$
\begin{aligned}
& b_{0}^{\infty}=\max \left\{\sqrt[4]{\alpha /\|V\|_{\infty}}, \sqrt[4]{\alpha /\|W\|_{\infty}},\right. \\
& b_{1}^{\infty}=\max \left\{\sqrt[4]{\|V\|_{\infty} / \alpha}, \sqrt[4]{\|W\|_{\infty} / \alpha}\right\} \\
& b_{2}^{\infty}=\max \left\{h\left(\sqrt{\|V\|_{\infty} / \alpha}\right), h\left(\sqrt{\|W\|_{\infty} / \alpha}\right)\right.
\end{aligned},
$$

where $h$ is defined in 2.6.

Then

$$
\mathcal{E}= \begin{cases}\mathcal{E}_{V} \cup \mathcal{E}_{W} & \text { for all } b \leq b_{0}^{\infty}, \\ \mathcal{E}_{\Sigma} & \text { for all } b>b_{1}^{\infty} .\end{cases}
$$

In addition, for every $b>b_{2}^{\infty}$ both the components of the solution remain bounded away from zero from below.

Remark 2.9. As $\mathcal{E}_{\Sigma} \subset \mathrm{Crit}_{C}(\Sigma)$, in particular, for $z \in \mathcal{E}_{\Sigma}$, we have

$$
0 \in \operatorname{Co}\left\{\lim _{j \rightarrow \infty} \nabla \Sigma\left(\xi_{j}\right): \xi_{j} \notin \mathcal{D} \text { and } \xi_{j} \rightarrow z\right\},
$$

where Co denotes the convex hull and $\mathcal{D}$ is any null set containing the set of points at which $\Sigma$ fails to be differentiable. This follows by a well known property of the Clarke subdifferential (see e.g. [11]).

Remark 2.10. Assume for a moment that system $\left(\sqrt{S_{z}}\right)$ admits a unique ground state solution, up to translations. Then, in light of formulas 4.15 it follows that $\Sigma$ is differentiable at $z, \partial_{C} \Sigma(z)=\{\nabla \Sigma(z)\}$, and hence, $\nabla \Sigma(z)=0$ provided that $z \in \mathcal{E}_{\Sigma}$. On the other hand, we point out that, in general, $\left(S_{z}\right)$ lacks uniqueness of ground state solutions. For instance, if $b=V(z)=W(z)=1$ and $U$ is the unique solution to $-\Delta U+U=U^{3}$ in $\mathbb{R}^{3}$, then the pairs $(\cos (\theta) U, \sin (\theta) U)$ with $0 \leq \theta \leq \pi / 2$ are all ground states solutions. In the case $b<1$, by the results of [18] the system has at least the scalar least energy solutions $(0, U)$ and $(U, 0)$. In the case $b>1$, we suspect that the system has a unique ground state solution. However, up to now, a proof seems out of reach.

\section{Proof of Theorem 2.1}

We will follow the arguments used in [13] for a single equation. Let $\gamma>0$ be such that

$$
\gamma<\frac{\alpha}{3 \sqrt{\max \{1, b\}}}
$$

For any $s, t \in \mathbb{R}$, set

$$
F_{\sharp}(s, t)= \begin{cases}\frac{1}{4}\left(s^{4}+2 b s^{2} t^{2}+t^{4}\right) & \text { if } s^{4}+2 b s^{2} t^{2}+t^{4} \leq \gamma^{2}, \\ \frac{\gamma}{2} \sqrt{s^{4}+2 b s^{2} t^{2}+t^{4}}-\frac{\gamma^{2}}{4} & \text { if } s^{4}+2 b s^{2} t^{2}+t^{4} \geq \gamma^{2} ;\end{cases}
$$


it follows that

$$
\nabla F_{\sharp}(s, t)= \begin{cases}\left(\left(s^{2}+b t^{2}\right) s,\left(t^{2}+b s^{2}\right) t\right) & \text { if } s^{4}+2 b s^{2} t^{2}+t^{4} \leq \gamma^{2}, \\ \gamma \frac{\left(\left(s^{2}+b t^{2}\right) s,\left(t^{2}+b s^{2}\right) t\right)}{\sqrt{s^{4}+2 b s^{2} t^{2}+t^{4}}} & \text { if } s^{4}+2 b s^{2} t^{2}+t^{4} \geq \gamma^{2} .\end{cases}
$$

It is easy to see that $F_{\sharp} \in C^{1}\left(\mathbb{R}^{2}\right)$. Let $B(z, r)$ be a ball with $z$ satisfying conditions (2.2)-2.3; we define

$$
G(x, s, t)=\chi(x) F(s, t)+(1-\chi(x)) F_{\sharp}(s, t)
$$

for a.e. $x \in \mathbb{R}^{3}$ and any $s, t \in \mathbb{R}$, where $\chi$ is the characteristic function of $B(z, r)$. In the light of the above definition, it follows that for every $(s, t)$ in $\mathbb{R}^{2}$,

$$
0 \leq 3 G(x, s, t)<\nabla G(x, s, t) \cdot(s, t) \quad \forall x \in B(z, r),
$$

and, for every $x \notin \overline{B(z, r)}$,

$$
0 \leq 2 G(x, s, t) \leq \nabla G(x, s, t) \cdot(s, t) \leq \frac{1}{k}\left[V(x) s^{2}+W(x) t^{2}\right] \quad \text { with } k>3 .
$$

We study the functional

$$
\tilde{J}_{\varepsilon}(u, v)=\frac{1}{2}\|u\|_{\varepsilon, V}^{2}+\frac{1}{2}\|v\|_{\varepsilon, W}^{2}-\int_{\mathbb{R}^{3}} G(x, u, v) .
$$

Note that $\tilde{J}_{\varepsilon}$ is of class $C^{1}$ on $\mathbb{H}$ and its critical points solve the system

$$
\begin{cases}-\varepsilon^{2} \Delta u+V(x) u=G_{u}(x, u, v) & \text { in } \mathbb{R}^{3} \\ -\varepsilon^{2} \Delta v+W(x) v=G_{v}(x, u, v) & \text { in } \mathbb{R}^{3}\end{cases}
$$

For each $\varepsilon>0$ fixed, we will find a critical point of $\tilde{J}_{\varepsilon}$ by applying the mountain pass theorem $([6])$, so that we define

$$
c_{\varepsilon}=\inf _{\gamma \in \Gamma} \sup _{t \in[0,1]} \tilde{J}_{\varepsilon}(\gamma(t))
$$

where $\Gamma=\left\{\gamma \in C([0,1], \mathbb{H}): \gamma(0)=(0,0), \tilde{J}_{\varepsilon}(\gamma(1))<0\right\}$. Arguing as in Lemma 2.1 of [13] and as in Lemma 3.2 of [18] one can prove that

$$
c_{\varepsilon}=\inf _{(u, v) \in \mathbb{H} \backslash\{(0,0)\}} \sup _{t \geq 0} \tilde{J}_{\varepsilon}(t u, t v) .
$$

Moreover, we will compare $c_{\varepsilon}$ with the level $\Sigma(z)$ (defined in (2.9)) of a ground state solution $\left(\varphi_{z}, \psi_{z}\right)$ of the limit system $\left(S_{z}\right)$. It is well known (see e.g. [10], [18]) that the functions $\varphi_{z}, \psi_{z}$ are radially symmetric, nonnegative, and decay exponentially to zero at infinity.

First of all, we show that $\tilde{J}_{\varepsilon}$ possesses suitably estimated critical values. 
Lemma 3.1. Assume 2.1. Then $\tilde{J}_{\varepsilon}$ has a nontrivial critical point $\left(u_{\varepsilon}, v_{\varepsilon}\right) \in \mathbb{H}$ such that

$$
\tilde{J}_{\varepsilon}\left(u_{\varepsilon}, v_{\varepsilon}\right) \leq \varepsilon^{3}(\Sigma(z)+o(1)),
$$

where $o(1) \rightarrow 0$ as $\varepsilon \rightarrow 0$. Moreover, there exists a positive constant $c_{0}$ such that

$$
\left\|u_{\varepsilon}\right\|_{\varepsilon, V}^{2}+\left\|v_{\varepsilon}\right\|_{\varepsilon, W}^{2} \leq c_{0} \varepsilon^{3} .
$$

Proof. Note that $(0,0)$ is a local minimum of $\tilde{J}_{\varepsilon}$, since $\tilde{J}_{\varepsilon}(u, v) \geq c\|(u, v)\|_{\mathbb{H}}^{2}$, provided that $\|(u, v)\|_{\mathbb{H}}$ is sufficiently small. Moreover, let $(\phi, \psi) \in \mathbb{H}$ with $\operatorname{supp}(\phi) \cup \operatorname{supp}(\psi) \subset$ $B(z, r)$ and observe that $\tilde{J}_{\varepsilon}(t(\phi, \psi)) \rightarrow-\infty$ as $t \rightarrow+\infty$. Then we can construct a Palais-Smale sequence at level $c_{\varepsilon}$ (defined in (3.5)). Conditions (3.2) and (3.3) imply that hypothesis ( $g 3$ ) in [13] is satisfied in our context, so that the compactness of Palais-Smale sequences can be recovered following the proof of Lemma 1.1 in [13]. By applying the mountain pass theorem $([\overline{6}])$, we get a nontrivial critical point $\left(u_{\varepsilon}, v_{\varepsilon}\right)$ at level $c_{\varepsilon}$. In order to show estimate (3.7), we need to consider a suitable pair of functions which models the concentration phenomenon. Define the functions

$$
u^{*}(x)=\eta(x) \varphi_{z}\left(\frac{x-z}{\varepsilon}\right), \quad v^{*}(x)=\eta(x) \psi_{z}\left(\frac{x-z}{\varepsilon}\right),
$$

where $\eta$ is a smooth function compactly supported in $B(z, r)$ and such that $\eta=1$ in a small neighborhood of $z$ and $\left(\varphi_{z}, \psi_{z}\right)$ is a ground state solution of problem $\left(S_{z}\right)$. From the definitions of $G(x, s, t)$ and $\eta(x)$ we deduce that $\tilde{J}_{\varepsilon}\left(t u^{*}, t v^{*}\right)=J_{\varepsilon}\left(t u^{*}, t v^{*}\right)$, so that it is easy to compute the supremum of $\tilde{J}_{\varepsilon}\left(t u^{*}, t v^{*}\right)$ for $t \geq 0$, and by using (3.6) we derive

$$
\tilde{J}_{\varepsilon}\left(u_{\varepsilon}, v_{\varepsilon}\right)=c_{\varepsilon} \leq \sup _{t \geq 0} \tilde{J}_{\varepsilon}\left(t u^{*}, t v^{*}\right)=\varepsilon^{3}[\Sigma(z)+o(1)],
$$

that is, (3.7) holds. Finally, using (3.7), the weak form of (3.4) tested with $\left(u_{\varepsilon}, v_{\varepsilon}\right)$ and (3.2), 3.3., it is possible to get also (3.8).

In the next proposition the asymptotic behavior outside $B(z, r)$ of the critical point $\left(u_{\varepsilon}, v_{\varepsilon}\right)$ found in Lemma 3.1 is studied.

Proposition 3.2. Assume 2.1] and that $z \in \mathbb{R}^{3}$ and $r>0$ satisfy conditions 2.2] and 2.3. Then for every $\delta>0$ there exists $\varepsilon_{\delta}>0$ such that

$$
\sup _{0<\varepsilon<\varepsilon_{\delta}} \sup _{x \in \mathbb{R}^{3} \backslash B(z, r)}\left(u_{\varepsilon}(x)+v_{\varepsilon}(x)\right)<\delta .
$$

Proof. Let us first prove that

$$
\lim _{\varepsilon \rightarrow 0} \sup _{x \in \partial B(z, r)}\left(u_{\varepsilon}(x)+v_{\varepsilon}(x)\right)=0 .
$$

We proceed by contradiction, assuming that there exist a sequence $\left\{\varepsilon_{n}\right\}$ converging to 0 and a sequence $\left\{x_{n}\right\} \subset \partial B(z, r)$ such that, for some positive constant $\beta$,

$$
u_{\varepsilon_{n}}\left(x_{n}\right)+v_{\varepsilon_{n}}\left(x_{n}\right) \geq \beta \quad \text { for all } n \geq 1 .
$$


Since $\partial B(z, r)$ is a compact set, we can assume that there exists a subsequence of $\left\{x_{n}\right\}$, still denoted by $\left\{x_{n}\right\}$, which converges to a point $x_{0} \in \partial B(z, r)$. Consider the scalings of $u_{\varepsilon_{n}}$ and $v_{\varepsilon_{n}}$ centered at $x_{n}$, that is,

$$
\phi_{n}(x)=u_{\varepsilon_{n}}\left(x_{n}+\varepsilon_{n} x\right), \quad \psi_{n}(x)=v_{\varepsilon_{n}}\left(x_{n}+\varepsilon_{n} x\right),
$$

which are critical points of the functional $\tilde{J}_{n}$ defined in $\mathbb{H}$ by

$$
\tilde{J}_{n}(u, v)=\frac{1}{2}\|u\|_{1, V\left(x_{n}+\varepsilon_{n} x\right)}^{2}+\frac{1}{2}\|v\|_{1, W\left(x_{n}+\varepsilon_{n} x\right)}^{2}-\int_{\mathbb{R}^{3}} G\left(x_{n}+\varepsilon_{n} x, u, v\right),
$$

so that the couple $\left(\phi_{n}, \psi_{n}\right)$ solves the system

$$
\left\{\begin{array}{l}
-\Delta \phi_{n}+V\left(x_{n}+\varepsilon_{n} x\right) \phi_{n}=G_{u}\left(x_{n}+\varepsilon_{n} x, \phi_{n}, \psi_{n}\right) \\
-\Delta \psi_{n}+W\left(x_{n}+\varepsilon_{n} x\right) \psi_{n}=G_{v}\left(x_{n}+\varepsilon_{n} x, \phi_{n}, \psi_{n}\right)
\end{array}\right.
$$

Notice that, by a simple change of scale, one can check that

$$
\tilde{J}_{n}\left(\phi_{n}, \psi_{n}\right)=\varepsilon_{n}^{-3} \tilde{J}_{\varepsilon_{n}}\left(u_{\varepsilon_{n}}, v_{\varepsilon_{n}}\right)
$$

From (3.8) we know that the sequences $\phi_{n}$ and $\psi_{n}$ are bounded in $H^{1}$; this, (3.12) and elliptic regularity estimates imply that $\phi_{n}$ and $\psi_{n}$ converge $C^{2}$ on compact sets to a couple $(\phi, \psi) \in \mathbb{H}$, which by 3.11 must be nontrivial. In addition, there exists a function $\xi \in L^{\infty}$ with $0 \leq \xi \leq 1$ such that $\chi\left(x_{n}+\varepsilon_{n} x\right)$ converges to $\xi$ weakly* in $L^{\infty}$. Then the pair $(\phi, \psi)$ is a solution of

$$
\left\{\begin{array}{l}
-\Delta \phi+V\left(x_{0}\right) \phi=\widehat{G}_{u}(x, \phi, \psi) \\
-\Delta \psi+W\left(x_{0}\right) \psi=\widehat{G}_{v}(x, \phi, \psi)
\end{array}\right.
$$

where $\widehat{G}(x, s, t)=\xi(x) F(s, t)+(1-\xi(x)) F_{\sharp}(s, t)$. The preceding system is the Euler equation of the functional

$$
J_{x_{0}}(u, v)=\frac{1}{2}\|u\|_{1, V\left(x_{0}\right)}^{2}+\frac{1}{2}\|v\|_{1, W\left(x_{0}\right)}^{2}-\int_{\mathbb{R}^{3}} \widehat{G}(x, u, v) .
$$

On the other hand, conditions (3.2) and (3.3) allow us to follow the same arguments of Lemma 2.2 in [13] to deduce that

$$
\liminf _{n \rightarrow \infty} J_{n}\left(\phi_{n}, \psi_{n}\right) \geq J_{x_{0}}(\phi, \psi)
$$

Indeed, consider the function

$$
\begin{aligned}
h_{n}= & \frac{1}{2}\left[\left|\nabla \phi_{n}\right|^{2}+\left|\nabla \psi_{n}\right|^{2}+V\left(x_{n}+\varepsilon_{n} x\right)\left|\phi_{n}\right|^{2}+W\left(x_{n}+\varepsilon_{n} x\right)\left|\psi_{n}\right|^{2}\right] \\
& -G\left(x_{n}+\varepsilon_{n} x, \phi_{n}, \psi_{n}\right) .
\end{aligned}
$$


Choosing $R>0$ sufficiently large, from the $C^{1}$ convergence of $\phi_{n}, \psi_{n}$ over compact sets, and since $\phi$ and $\psi$ belong to $H^{1}$, we have, for every $\delta>0$ fixed,

$$
\lim _{n \rightarrow \infty} \int_{B_{R}} h_{n} \geq J_{x_{0}}(\phi, \psi)-\delta,
$$

where $B_{R}$ stands for $B(0, R)$. Moreover, taking $\eta_{R}$ a smooth cut-off function such that $\eta_{R}=0$ on $B_{R-1}$ and $\eta_{R}=1$ on $\mathbb{R}^{3} \backslash B_{R}$, and using as test function in 3.12 $w=$ $\eta_{R}\left(\phi_{n}, \psi_{n}\right)$, we obtain

$$
\liminf _{n \rightarrow \infty} \int_{\mathbb{R}^{3} \backslash B_{R}} h_{n} \geq-\delta,
$$

yielding (3.14). Since $(\phi, \psi)$ is a critical point of $J_{x_{0}}$ we have

$$
J_{x_{0}}(\phi, \psi)=\max _{t \geq 0} J_{x_{0}}(t(\phi, \psi))
$$

Moreover, $F(s, t) \geq F_{\sharp}(s, t)$, so that $\widehat{G}(x, s, t) \leq F(s, t)$, which, together with 3.15), implies that

$$
J_{x_{0}}(\phi, \psi) \geq \inf _{(u, v) \in \mathbb{H}} \sup _{t \geq 0} I_{x_{0}}(t(u, v))=\Sigma\left(x_{0}\right) .
$$

From assumptions 2.2, 2.3 it follows that $V\left(x_{0}\right)>V_{0}$ and $W\left(x_{0}\right)>W_{0}$; this means that $\Sigma\left(x_{0}\right)>\Sigma(z)$, where $\Sigma(z)$ is defined in 2.9. This, 3.13, 3.14) and 3.16 yield

$$
\Sigma(z)<J_{x_{0}}(\phi, \psi) \leq \liminf _{n \rightarrow \infty} J_{n}\left(\phi_{n}, \psi_{n}\right) \leq \Sigma(z),
$$

which is a contradiction, proving 3.10.

We are now ready to conclude the proof of the result. Fix $\delta>0$; from 3.10 it follows that there exists $\varepsilon_{\delta}>0$ such that $0 \leq u_{\varepsilon}(x)<\delta$ and $0 \leq v_{\varepsilon}(x)<\delta$ for any $x \in \partial B(z, r)$ and $\varepsilon \in\left(0, \varepsilon_{\delta}\right)$. It follows that $\left(u_{\varepsilon}-\delta\right)^{+}=0$ and $\left(v_{\varepsilon}-\delta\right)^{+}=0$ on $\partial B(z, r)$ and hence we can choose

$$
\phi_{\varepsilon}=\left(u_{\varepsilon}-\delta\right)^{+} \chi_{\{|x-z|>r\}} \in H^{1}, \quad \psi_{\varepsilon}=\left(v_{\varepsilon}-\delta\right)^{+} \chi_{\{|x-z|>r\}} \in H^{1}
$$

as test functions for system (3.4). By multiplying and integrating over $\mathbb{R}^{3}$, we obtain

$$
\begin{aligned}
& \int_{\mathbb{R}^{3} \backslash B(z, r)}\left(\varepsilon^{2}\left|\nabla\left(u_{\varepsilon}-\delta\right)^{+}\right|^{2}+V(x) u_{\varepsilon}\left(u_{\varepsilon}-\delta\right)^{+}-G_{u}\left(x, u_{\varepsilon}, v_{\varepsilon}\right)\left(u_{\varepsilon}-\delta\right)^{+}\right) \\
& \quad+\int_{\mathbb{R}^{3} \backslash B(z, r)}\left(\varepsilon^{2}\left|\nabla\left(v_{\varepsilon}-\delta\right)^{+}\right|^{2}+W(x) v_{\varepsilon}\left(v_{\varepsilon}-\delta\right)^{+}-G_{v}\left(x, u_{\varepsilon}, v_{\varepsilon}\right)\left(v_{\varepsilon}-\delta\right)^{+}\right)=0 .
\end{aligned}
$$

Note that, since we can write

$$
G_{u}\left(x, u_{\varepsilon}, v_{\varepsilon}\right)= \begin{cases}\frac{G_{u}\left(x, u_{\varepsilon}, v_{\varepsilon}\right)}{u_{\varepsilon}}\left[\left(u_{\varepsilon}-\delta\right)+\delta\right] & \text { if } u_{\varepsilon}(x)>0, \\ 0 & \text { if } u_{\varepsilon}(x)=0,\end{cases}
$$


and

$$
G_{v}\left(x, u_{\varepsilon}, v_{\varepsilon}\right)= \begin{cases}\frac{G_{v}\left(x, u_{\varepsilon}, v_{\varepsilon}\right)}{v_{\varepsilon}}\left[\left(v_{\varepsilon}-\delta\right)+\delta\right] & \text { if } v_{\varepsilon}(x)>0, \\ 0 & \text { if } v_{\varepsilon}(x)=0,\end{cases}
$$

the preceding identity turns into

$$
\begin{aligned}
\int_{\mathbb{R}^{3} \backslash B(z)}\left(\varepsilon^{2}\left|\nabla\left(u_{\varepsilon}-\delta\right)^{+}\right|^{2}+\Upsilon_{\varepsilon}(x)\left|\left(u_{\varepsilon}-\delta\right)^{+}\right|^{2}+\Upsilon_{\varepsilon}(x) \delta\left(u_{\varepsilon}-\delta\right)^{+}\right) \\
\quad+\int_{\mathbb{R}^{3} \backslash B(z)}\left(\varepsilon^{2}\left|\nabla\left(v_{\varepsilon}-\delta\right)^{+}\right|^{2}+\Lambda_{\varepsilon}(x)\left|\left(v_{\varepsilon}-\delta\right)^{+}\right|^{2}+\Lambda_{\varepsilon}(x) \delta\left(v_{\varepsilon}-\delta\right)^{+}\right)=0,
\end{aligned}
$$

where we have set

$$
\begin{aligned}
& \Upsilon_{\varepsilon}(x)=V(x)-\gamma \frac{u_{\varepsilon}^{2}(x)+b v_{\varepsilon}^{2}(x)}{\sqrt{u_{\varepsilon}^{4}(x)+2 b u_{\varepsilon}^{2}(x) v_{\varepsilon}^{2}(x)+v_{\varepsilon}^{4}(x)}}, \\
& \Lambda_{\varepsilon}(x)=W(x)-\gamma \frac{v_{\varepsilon}^{2}(x)+b u_{\varepsilon}^{2}(x)}{\sqrt{u_{\varepsilon}^{4}(x)+2 b u_{\varepsilon}^{2}(x) v_{\varepsilon}^{2}(x)+v_{\varepsilon}^{4}(x)}} .
\end{aligned}
$$

By (3.1), it is easy to show that $\Upsilon_{\varepsilon}(x) \geq 2 \alpha / 3$ and $\Lambda_{\varepsilon}(x) \geq 2 \alpha / 3$ for all $x$ with $u_{\varepsilon}(x)>0$ or $v_{\varepsilon}(x)>0$, which implies that $\left(u_{\varepsilon}(x)-\delta\right)^{+}=0$ and $\left(u_{\varepsilon}(x)-\delta\right)^{+}=0$ for every $x \notin B(z, r)$ and every $0<\varepsilon<\varepsilon_{\delta}$, yielding the assertion.

When proving Theorem 2.1 we will use Theorem 2.9 of [18] which gives a necessary condition for the existence of a vector ground state (that is, a ground state $(u, v)$ with $u>0$ and $v>0$ ) for an autonomous system. Here, for the reader's convenience, we briefly sketch the proof in the presence of two different constant potentials.

Proposition 3.3. Let $\kappa_{1}, \kappa_{2}>0$ and $(u, v) \in H^{1} \times H^{1}$ be a least energy solution of the system

$$
\left\{\begin{aligned}
-\Delta u+\kappa_{1} u=u^{3}+b v^{2} u & & \text { in } \mathbb{R}^{3} \\
-\Delta v+\kappa_{2} v=v^{3}+b u^{2} v & & \text { in } \mathbb{R}^{3}
\end{aligned}\right.
$$

Define

$$
b_{0}=\max \left\{\sqrt[4]{\kappa_{1} / \kappa_{2}}, \sqrt[4]{\kappa_{2} / \kappa_{1}}\right\}, \quad b_{1}=\max \left\{h\left(\sqrt{\kappa_{1} / \kappa_{2}}\right), h\left(\sqrt{\kappa_{2} / \kappa_{1}}\right)\right\},
$$

where $h(s)$ is defined in (2.6). Then the following facts hold:

(a) if $b<b_{0}$ then either $u \equiv 0$ and $v \neq \equiv 0$, or $u \neq 0$ and $v \equiv 0$,

(b) if $b>b_{1}$ then $u \not \equiv 0$ and $v \not \equiv 0$.

Proof. Suppose that $(u, v)$ is a vector ground state of 3.18 and assume, without loss of generality, that $0<\kappa_{2} \leq \kappa_{1}$. Consider the functions

$$
\bar{u}(x)=\frac{1}{\sqrt{k_{1}}} u\left(\frac{x}{\sqrt{k_{1}}}\right), \quad \bar{v}(x)=\frac{1}{\sqrt{k_{1}}} v\left(\frac{x}{\sqrt{k_{1}}}\right) .
$$


Then the above system becomes

$$
\begin{cases}-\Delta \bar{u}+\bar{u}=\bar{u}^{3}+b \bar{v}^{2} \bar{u} & \text { in } \mathbb{R}^{3} \\ -\Delta \bar{v}+\omega^{2} \bar{v}=\bar{v}^{3}+b \bar{u}^{2} \bar{v} & \text { in } \mathbb{R}^{3}\end{cases}
$$

where we have set $\omega^{2}=\kappa_{2} / \kappa_{1} \leq 1$. Then conclusion (a) follows by applying [18, Theorem 2.9], whereas conclusion (b) can be obtained by arguing as in the proofs of [18, Theorems 2.3, 2.8] (see Remark 2.11 therein).

Proof of Theorem 2.1. By Proposition 3.2, taking into account the definition of $G$, the pair $\left(u_{\varepsilon}, v_{\varepsilon}\right) \neq(0,0)$ is a solution of $\left(S_{\varepsilon}\right)$. From elliptic regularity theory it follows that $u_{\varepsilon}, v_{\varepsilon}$ are nonnegative $C^{2}$ functions. Let $\xi_{\varepsilon}$ be a local maximum point of the function $u_{\varepsilon}(x)+v_{\varepsilon}(x)$. Then

$$
\begin{aligned}
0 \leq-\Delta\left(u_{\varepsilon}+v_{\varepsilon}\right)\left(\xi_{\varepsilon}\right)= & -V\left(\xi_{\varepsilon}\right) u_{\varepsilon}\left(\xi_{\varepsilon}\right)-W\left(\xi_{\varepsilon}\right) v_{\varepsilon}\left(\xi_{\varepsilon}\right) \\
& +\left(u_{\varepsilon}^{2}\left(\xi_{\varepsilon}\right)+b v_{\varepsilon}^{2}\left(\xi_{\varepsilon}\right)\right) u_{\varepsilon}\left(\xi_{\varepsilon}\right)+\left(v_{\varepsilon}^{2}\left(\xi_{\varepsilon}\right)+b u_{\varepsilon}^{2}\left(\xi_{\varepsilon}\right)\right) v_{\varepsilon}\left(\xi_{\varepsilon}\right) .
\end{aligned}
$$

Using (2.1), there exists a positive radius $\sigma$, independent of $\varepsilon$, such that

$$
\left(u_{\varepsilon}+v_{\varepsilon}\right)\left(\xi_{\varepsilon}\right) \geq \sigma
$$

Let us first prove (2.4) of conclusion (i) in Theorem 2.1 arguing by contradiction. More precisely, consider $\varepsilon_{n} \rightarrow 0$ and $x_{n} \in B(z, r)$ a local maximum point of $u_{\varepsilon_{n}}+v_{\varepsilon_{n}}$. Let $x_{n} \rightarrow x^{*} \in \bar{B}(z, r)$, and assume that both $V\left(x^{*}\right)>V_{0}$ and $W\left(x^{*}\right)>W_{0}$. Then we can consider the sequences $\phi_{n}(x)=u_{\varepsilon_{n}}\left(x_{n}+\varepsilon_{n} x\right), \psi_{n}(x)=v_{\varepsilon_{n}}\left(x_{n}+\varepsilon_{n} x\right)$ and the limit $(\phi, \psi)$, a critical point of the limit functional $I_{x^{*}}$. First, note that $(\phi, \psi) \neq(0,0)$ thanks to 3.20; moreover, by virtue of the inequalities $V\left(x^{*}\right)>V_{0}$ and $W\left(x^{*}\right)>W_{0}$, the critical level $I_{x^{*}}(\phi, \psi)$ can be compared with $\Sigma(z)$, yielding again a contradiction. Then, in order to prove conclusion (i) of Theorem 2.1, it is only left to show the uniqueness of the maximum point of the function $u_{\varepsilon}+v_{\varepsilon}$. Assume by contradiction that there exist a sequence $\left\{\varepsilon_{n}\right\}$ converging to zero and two local maxima $x_{n}^{1}, x_{n}^{2} \in \bar{B}(z, r)$, which both satisfy 3.20 . We consider the sequences

$$
\phi_{n}(x)=u_{\varepsilon_{n}}\left(x_{n}^{1}+\varepsilon_{n} x\right) \text { and } \quad \psi_{n}(x)=v_{\varepsilon_{n}}\left(x_{n}^{1}+\varepsilon_{n} x\right) .
$$

Arguing as before, we show that the couple $\left(\phi_{n}, \psi_{n}\right)$ converges in the $C^{2}$ sense over compact sets to a solution $(\phi, \psi)$ of $\left(S_{z}\right)$ with $z=x_{1}$ and $V\left(x^{1}\right)=V_{0}$ and $W\left(x^{1}\right)=$ $W_{0}$. From $[3.20$ ) we see that $(\phi, \psi) \neq(0,0)$ and from [10] we deduce that $(\phi, \psi)$ are nonnegative, radially symmetric functions. Then the sum $\phi+\psi$ has a local nondegenerate maximum point, which, up to translations, is located at the origin. This fact and the $C^{2}$ convergence of $\phi_{n}+\psi_{n}$ imply that $x_{n}=\left(x_{n}^{2}-x_{n}^{1}\right) / \varepsilon_{n} \rightarrow \infty$. Then we can argue as in the proof of (3.14) to get a contradiction. Indeed, we consider the function

$$
h_{n}=\frac{1}{2}\left[\left|\nabla \phi_{n}\right|^{2}+\left|\nabla \psi_{n}\right|^{2}+V\left(x_{n}^{1}+\varepsilon_{n} x\right) \phi_{n}^{2}+W\left(x_{n}^{1}+\varepsilon_{n} x\right) \psi_{n}^{2}\right]-F\left(\phi_{n}, \psi_{n}\right) .
$$


For every $\delta$ we can choose $R>0$ and $n_{0}$ sufficiently large such that $B_{R} \cap B_{R}\left(x_{n}\right)=\emptyset$ for every $n \geq n_{0}$ and

$$
\lim _{n \rightarrow \infty} \int_{B_{R}(0)} h_{n} \geq I_{x^{1}}(\phi, \psi)-\delta
$$

Moreover,

$$
\begin{aligned}
\lim _{n \rightarrow \infty} \int_{B_{R}\left(x_{n}\right)} h_{n}=\frac{1}{2} \lim _{n \rightarrow \infty}\left\{\int _ { B _ { R } } \left[\left|\nabla \bar{\phi}_{n}\right|^{2}\right.\right. & +\left|\nabla \bar{\psi}_{n}\right|^{2}+V\left(x_{n}^{2}+\varepsilon_{n} x_{n}\right) \bar{\phi}_{n}^{2} \\
& \left.\left.+W\left(x_{n}^{2}+\varepsilon_{n} x_{n}\right) \bar{\psi}_{n}^{2}\right]-\int_{B_{R}} F\left(\bar{\phi}_{n}, \bar{\psi}_{n}\right)\right\}
\end{aligned}
$$

where we have put $\bar{\phi}_{n}(y)=\phi_{n}\left(y+x_{n}\right), \bar{\psi}_{n}(y)=\psi_{n}\left(y+x_{n}\right)$. As $V\left(x^{1}\right)=V\left(x^{2}\right)=V_{0}$ and $W\left(x^{1}\right)=W\left(x^{2}\right)=W_{0}$, we get

$$
\lim _{n \rightarrow \infty} \int_{B_{R}\left(x_{n}\right)} h_{n} \geq I_{x^{2}}(\bar{\phi}, \bar{\psi})-\delta=I_{x^{1}}(\phi, \psi)-\delta .
$$

Then, arguing as in the proof of (3.14), we get

$$
\liminf _{n \rightarrow \infty} J_{n}\left(\phi_{n}, \psi_{n}\right) \geq 2 \Sigma\left(x^{1}\right)=2 \Sigma(z)
$$

which contradicts 3.7 .

In order to prove the exponential decay, notice that, by Proposition 3.2 $u_{\varepsilon}$ and $v_{\varepsilon}$ decay to zero at infinity, uniformly with respect to $\varepsilon$. Hence we find $\rho>0, \Theta \in(0, \sqrt{\alpha})$ and $\varepsilon_{0}>0$ such that $u_{\varepsilon}^{2}+b v_{\varepsilon}^{2} \leq \alpha-\Theta^{2}$ and $v_{\varepsilon}^{2}+b u_{\varepsilon}^{2} \leq \alpha-\Theta^{2}$ for all $\left|x-x_{\varepsilon}\right|>\varepsilon \rho$ and $0<\varepsilon<\varepsilon_{0}$. Set

$$
\xi_{\rho}(x)=M_{\rho} e^{-\Theta\left(\left|x-x_{\varepsilon}\right| / \varepsilon-\rho\right)}, \quad M_{\rho}=\sup _{\left(0, \varepsilon_{0}\right)} \max _{|x|=\rho}\left(u_{\varepsilon}+v_{\varepsilon}\right),
$$

and introduce the set $\mathcal{A}=\bigcup_{R>\rho} D_{R}$, where, for any $R>\rho$,

$$
D_{R}=\left\{\rho<|x|<R: u_{\varepsilon}(x)+v_{\varepsilon}(x)>\xi_{\rho}(x) \text { for some } \varepsilon \in\left(0, \varepsilon_{0}\right)\right\} .
$$

Assume by contradiction that $\mathcal{A} \neq \emptyset$. Then there exist $R_{*}>\rho$ and $\varepsilon_{*} \in\left(0, \varepsilon_{0}\right)$ with

$$
\begin{aligned}
\varepsilon^{2} \Delta\left(\xi_{\rho}-u_{\varepsilon_{*}}-v_{\varepsilon_{*}}\right) & \leq\left[\Theta^{2}-\frac{2 \varepsilon \Theta}{\left|x-x_{\varepsilon}\right|}\right] \xi_{\rho}-\Theta^{2} u_{\varepsilon_{*}}-\Theta^{2} v_{\varepsilon_{*}} \\
& \leq \Theta^{2}\left(\xi_{\rho}-u_{\varepsilon_{*}}-v_{\varepsilon_{*}}\right)<0 \text { in } D_{R} \text { for all } R \geq R_{*} .
\end{aligned}
$$

Hence, by the maximum principle, we get

$$
\xi_{\rho}-u_{\varepsilon_{*}}-v_{\varepsilon_{*}} \geq \min \left\{\min _{|x|=\rho}\left(\xi_{\rho}-u_{\varepsilon_{*}}-v_{\varepsilon_{*}}\right), \min _{|x|=R}\left(\xi_{\rho}-u_{\varepsilon_{*}}-v_{\varepsilon_{*}}\right)\right\}
$$

in $D_{R}$ for all $R \geq R_{*}$. Letting $R \rightarrow \infty$ and recalling the definition of $\xi_{\rho}$ yields

$$
\xi_{\rho}-u_{\varepsilon_{*}}-v_{\varepsilon_{*}} \geq \min \left\{\min _{|x|=\rho}\left(\xi_{\rho}-u_{\varepsilon_{*}}-v_{\varepsilon_{*}}\right), 0\right\} \geq 0 \quad \text { in } \bigcup_{R \geq R_{*}} D_{R}
$$


In turn, $u_{\varepsilon_{*}}(x)+v_{\varepsilon_{*}}(x) \leq \xi_{\rho}(x)$ for all $x$ in $\bigcup_{R \geq R_{*}} D_{R}$, which yields a contradiction. Hence $\mathcal{A}=\varnothing$, and the desired exponential decay follows.

Now we prove conclusion (ii) of Theorem 2.1. Once again, let us set $\left(\phi_{\varepsilon}, \psi_{\varepsilon}\right)=$ $\left(u_{\varepsilon}\left(x_{\varepsilon}+\varepsilon x\right), v_{\varepsilon}\left(x_{\varepsilon}+\varepsilon x\right)\right)$. Note that (3.8) gives $\left\|\left(\phi_{\varepsilon}, \psi_{\varepsilon}\right)\right\|_{\mathbb{H}} \leq C$ and the pair $\left(\phi_{\varepsilon}, \psi_{\varepsilon}\right)$ solves

$$
\begin{cases}-\Delta \phi_{\varepsilon}+V\left(x_{\varepsilon}+\varepsilon x\right) \phi_{\varepsilon}=\phi_{\varepsilon}^{3}+b \psi_{\varepsilon}^{2} \phi_{\varepsilon} & \text { in } \mathbb{R}^{3}, \\ -\Delta \psi_{\varepsilon}+W\left(x_{\varepsilon}+\varepsilon x\right) \psi_{\varepsilon}=\psi_{\varepsilon}^{3}+b \phi_{\varepsilon}^{2} \psi_{\varepsilon} & \text { in } \mathbb{R}^{3} .\end{cases}
$$

From the conclusion (i) we deduce that $x_{\varepsilon}$ converges to $p$, with $V(p)=V_{0}$ and $W(p)=$ $W_{0}$, and $\left(\phi_{\varepsilon}, \psi_{\varepsilon}\right)$ converges to $(\phi, \psi)$, a least energy solution of (3.18) with $\kappa_{1}=V_{0}$ and $\kappa_{2}=W_{0}$. Then, if $b<b_{0}$, in the light of Proposition 3.3, either $\phi \equiv 0$ or $\psi \equiv 0$. Since $\phi_{\varepsilon}$ and $\psi_{\varepsilon}$ converge uniformly over compact sets, we see that either $u_{\varepsilon}\left(x_{\varepsilon}\right)=\phi_{\varepsilon}(0) \rightarrow 0$ or $v_{\varepsilon}\left(x_{\varepsilon}\right)=\psi_{\varepsilon}(0) \rightarrow 0$. Similarly, if $b>b_{1}$, in the light of Proposition $3.3, \phi \neq 0$ and $\psi \neq 0$, and the assertion follows.

Remark 3.4. In the previous theorem we have proved that the least energy solution $\left(u_{\varepsilon}, v_{\varepsilon}\right)$ converges (up to scalings) to a least energy (by $(3.7)$ ) solution $(\phi, \psi)$ of

$$
\left\{\begin{array}{l}
-\Delta \phi+V_{0} \phi=\phi^{3}+b \psi^{2} \phi \\
-\Delta \psi+W_{0} \psi=\psi^{3}+b \phi^{2} \psi
\end{array}\right.
$$

Moreover, for $b<b_{0}$, one of $\phi, \psi$ is necessarily zero; so that $(\phi, \psi)$ is actually either $(\phi, 0)$ or $(0, \psi)$, with $\phi$ (respectively $\psi)$ the unique least energy solution of $-\Delta \phi+V_{0} \phi$ $=\phi^{3}$ (respectively $-\Delta \psi+W_{0} \psi=\psi^{3}$ ). Thus, if $V_{0}<W_{0}$, the least scalar energy solution of 3.23 is $(\phi, 0)$, yielding $v_{\varepsilon}\left(x_{\varepsilon}\right) \rightarrow 0$. Otherwise, if $W_{0}<V_{0}, u_{\varepsilon}\left(x_{\varepsilon}\right) \rightarrow 0$.

Proof of Theorem 2.3 It suffices to run through the various steps of the proof of Theorem 2.1 up to formula (3.16). Now, in order to obtain (3.17) we can use hypothesis 2.10) instead of 2.2, 2.3 to get directly

$$
\Sigma(z)<\Sigma\left(x_{0}\right) \leq J_{x_{0}}(\phi, \psi) \leq \liminf _{n \rightarrow \infty} J_{n}\left(\phi_{n}, \psi_{n}\right) \leq \Sigma(z),
$$

as $x_{0} \in \partial B(z, r)$ and $z \in B(z, r)$, yielding the desired contradiction and thus eventually proving Proposition 3.2 . If $x_{\varepsilon}$ is the sequence of maximum points, then $\Sigma\left(x_{\varepsilon}\right) \rightarrow \Sigma_{0}$, as otherwise one would get a contradiction similar to the one above. The dichotomy and the exponential decay can be proved exactly as in the proof of Theorem 2.1.

\section{Proof of Theorem 2.8}

To prove Theorem 2.8 the following preliminary lemma will be useful.

Lemma 4.1. Assume that $V, W \in C^{1}\left(\mathbb{R}^{3}\right)$ satisfy 2.13). If $z \in \mathcal{E}$, then

$$
\gamma_{1}(z) \nabla V(z)+\gamma_{2}(z) \nabla W(z)=0
$$

for some $\gamma_{1}(z) \geq 0, \gamma_{2}(z) \geq 0$, one of them being nontrivial. 
Proof. Let $z \in \mathcal{E}, \varepsilon_{n}$ a sequence converging to zero and $\left(u_{\varepsilon_{n}}, v_{\varepsilon_{n}}\right)$ a solution of problem $\left[S_{\varepsilon}\right.$ that satisfies the properties in Definition 2.6 Define $\varphi_{n}(x)=u_{\varepsilon_{n}}\left(z+\varepsilon_{n} x\right), \psi_{n}(x)=$ $v_{\varepsilon_{n}}\left(z+\varepsilon_{n} x\right)$ and consider the lagrangian $\mathcal{L}: \mathbb{R}^{3} \times \mathbb{R} \times \mathbb{R} \times \mathbb{R}^{3} \times \mathbb{R}^{3} \rightarrow \mathbb{R}$ defined as

$\mathcal{L}\left(x, s_{1}, s_{2}, \xi_{1}, \xi_{2}\right)=\frac{\left|\xi_{1}\right|^{2}+\left|\xi_{2}\right|^{2}}{2}+V\left(z+\varepsilon_{n} x\right) \frac{s_{1}^{2}}{2}+W\left(z+\varepsilon_{n} x\right) \frac{s_{2}^{2}}{2}-\frac{s_{1}^{4}+2 b s_{1}^{2} s_{2}^{2}+s_{2}^{4}}{4}$.

By the Pucci-Serrin identity for systems [22, see §5], we have

$$
\begin{aligned}
\sum_{i, \ell=1}^{3} \int_{\mathbb{R}^{3}} \partial_{i} \boldsymbol{h}^{\ell} \partial_{i} \psi_{n} \partial_{\ell} \psi_{n} & +\sum_{i, \ell=1}^{3} \int_{\mathbb{R}^{3}} \partial_{i} \boldsymbol{h}^{\ell} \partial_{i} \varphi_{n} \partial_{\ell} \varphi_{n} \\
= & \int_{\mathbb{R}^{3}} \operatorname{div} \boldsymbol{h} \mathcal{L}\left(x, \varphi_{n}, \psi_{n}, \nabla \varphi_{n}, \nabla \psi_{n}\right) \\
& +\frac{1}{2} \int_{\mathbb{R}^{3}} \varepsilon_{n} \boldsymbol{h} \cdot\left[\nabla V\left(z+\varepsilon_{n} x\right) \varphi_{n}^{2}+\nabla W\left(z+\varepsilon_{n} x\right) \psi_{n}^{2}\right]
\end{aligned}
$$

for all $h \in C_{\mathrm{c}}^{1}\left(\mathbb{R}^{3}, \mathbb{R}^{3}\right)$. Choose, for any $\lambda>0$,

$$
\boldsymbol{h}_{j}: \mathbb{R}^{3} \rightarrow \mathbb{R}^{3}, \quad \boldsymbol{h}_{j}^{\ell}(x)=\left\{\begin{array}{ll}
\Upsilon(\lambda x) & \text { if } j=\ell, \\
0 & \text { if } j \neq \ell,
\end{array} \quad \ell=1,2,3,\right.
$$

$\Upsilon \in C_{\mathrm{c}}^{1}\left(\mathbb{R}^{3}\right)$, where $\Upsilon(x)=1$ if $|x| \leq 1$ and $\Upsilon(x)=0$ if $|x| \geq 2$. Then, for $j=1,2,3$,

$$
\begin{aligned}
\sum_{i=1}^{3} \int_{\mathbb{R}^{3}} \lambda \partial_{i} \Upsilon(\lambda x) \partial_{i} \psi_{n} & \partial_{j} \psi_{n}+\sum_{i=1}^{3} \int_{\mathbb{R}^{3}} \lambda \partial_{i} \Upsilon(\lambda x) \partial_{i} \varphi_{n} \partial_{j} \varphi_{n} \\
= & \int_{\mathbb{R}^{3}} \lambda \partial_{j} \Upsilon(\lambda x) \mathcal{L}\left(x, \varphi_{n}, \psi_{n}, \nabla \varphi_{n}, \nabla \psi_{n}\right) \\
& +\frac{1}{2} \int_{\mathbb{R}^{3}} \varepsilon_{n} \Upsilon(\lambda x)\left[\partial_{j} V\left(z+\varepsilon_{n} x\right) \varphi_{n}^{2}+\partial_{j} W\left(z+\varepsilon_{n} x\right) \psi_{n}^{2}\right]
\end{aligned}
$$

By the arbitrariness of $\lambda>0$, letting $\lambda \rightarrow 0$ and keeping $j$ fixed, we obtain

$$
\int_{\mathbb{R}^{3}}\left[\partial_{j} V\left(z+\varepsilon_{n} x\right) \varphi_{n}^{2}+\partial_{j} W\left(z+\varepsilon_{n} x\right) \psi_{n}^{2}\right]=0, \quad j=1,2,3 .
$$

By assumption 2.13, there exists a positive constant $\beta_{1}$ such that, for all $x \in \mathbb{R}^{3}$ and $j \geq 1$, we get $\left|\nabla V\left(z+\varepsilon_{n} x\right)\right| \leq \beta_{1} e^{\gamma \varepsilon_{n}|x|}$ and $\left|\nabla W\left(z+\varepsilon_{n} x\right)\right| \leq \beta_{1} e^{\gamma \varepsilon_{n}|x|}$, so that, invoking the uniform exponential decay of $\varphi_{n}$ and $\psi_{n}$, letting $n \rightarrow \infty$ in the above identity, we obtain

$$
\int_{\mathbb{R}^{3}}\left(\partial_{j} V(z) \varphi_{z}^{2}+\partial_{j} W(z) \psi_{z}^{2}\right)=0, \quad j=1,2,3,
$$

where $\left(\varphi_{z}, \psi_{z}\right) \neq(0,0)$ is a least energy solution of $\left(S_{z}\right)$.

Therefore 4.1 holds with $\gamma_{1}(z)=\left\|\varphi_{z}\right\|_{2}^{2}$ and $\gamma_{2}(z)=\left\|\psi_{z}\right\|_{2}^{2}$. 
Proof of Theorem 2.8 First, we will show that $\Sigma$ is a continuous function. Recall from [18. Lemma 3.1] that, for every $\xi \in \mathbb{R}^{3}$ and $w \in H^{1} \times H^{1}$ with $w \neq(0,0)$, there exists a unique $\theta(w, \xi)>0$ such that $\theta(w, \xi) w \in \mathcal{N}_{\xi}$ (defined in (2.8)); the map $w \mapsto$ $\theta(w, \xi)$ is continuous and $w \mapsto \theta(w, \xi) w$ is a homeomorphism of the unit sphere of $H^{1} \times H^{1}$ on $\mathcal{N}_{\xi}$. In order to prove that $\Sigma$ defined in 2.9 is continuous, first assume that the potentials $V(x), W(x)$ are positive constants $V, W \in \mathbb{R}^{+}$. Following the lines of [23], we show the continuity of the map $(V, W) \mapsto c(V, W)$, where $c(V, W)$ is the mountain pass level of the functional $I_{V, W}: H^{1} \times H^{1} \rightarrow \mathbb{R}$ defined by

$$
I_{V, W}(u, v)=\frac{1}{2} \int_{\mathbb{R}^{3}}\left[|\nabla u|^{2}+|\nabla v|^{2}+V u^{2}+W v^{2}\right]-\int_{\mathbb{R}^{3}} F(u, v) .
$$

The following equalities hold (see Lemma 3.1 in [18]):

$$
c(V, W)=\inf _{H^{1} \times H^{1} \backslash(0,0)} \max _{t \geq 0} I_{V, W}(t u, t v)=\inf _{\mathcal{N}_{V, W}} I_{V, W},
$$

where $\mathcal{N}_{V, W}$ is the Nehari manifold associated to $I_{V, W}$. Note that 4.3 implies that proving the continuity of the map $c(V, W)$ is equivalent to showing the continuity of the map $(V, W) \mapsto \Sigma(V, W)$. Let us first show that

$$
\lim _{\eta \rightarrow 0} c(V+\eta, W+\eta)=c(V, W) .
$$

It is readily seen that the following monotonicity property holds:

$$
V_{1}>V_{2}, W_{1}>W_{2} \Rightarrow c\left(V_{1}, W_{1}\right) \geq c\left(V_{2}, W_{2}\right) .
$$

By virtue of 4.5, we get

$$
\lim _{\eta \rightarrow 0^{-}} c(V+\eta, W+\eta)=: c^{-} \leq c(V, W) .
$$

Let $\eta_{h} \rightarrow 0^{-}$and $\delta_{h} \rightarrow 0^{+}$as $h \rightarrow \infty$. By the definition of $c(V+\eta, W+\eta)$ and (4.3), and since the map $\theta$ induces a homeomorphism of the unit sphere of $H^{1} \times H^{1}$ on $\mathcal{N}_{V+\eta_{h}, W+\eta_{h}}$, there exists $\left(u_{h}, v_{h}\right) \in H^{1} \times H^{1}$ such that

$$
\begin{gathered}
\left\|\nabla u_{h}\right\|_{2}^{2}+\left\|\nabla v_{h}\right\|_{2}^{2}+\left\|u_{h}\right\|_{2}^{2}+\left\|v_{h}\right\|_{2}^{2}=1, \\
\max _{t \geq 0} I_{V+\eta_{h}, W+\eta_{h}}\left(t u_{h}, t v_{h}\right) \leq c\left(V+\eta_{h}, W+\eta_{h}\right)+\delta_{h} .
\end{gathered}
$$

We will first show that $\theta\left(u_{h}, v_{h}\right)$, given by

$$
\theta\left(u_{h}, v_{h}\right)=\sqrt{\frac{\left\|\nabla u_{h}\right\|_{2}^{2}+\left\|\nabla v_{h}\right\|_{2}^{2}+V\left\|u_{h}\right\|_{2}^{2}+W\left\|v_{h}\right\|_{2}^{2}}{\left\|u_{h}\right\|_{4}^{4}+\left\|v_{h}\right\|_{4}^{4}+2 b\left\|u_{h} v_{h}\right\|_{2}^{2}}},
$$

remains bounded. Arguing by contradiction, suppose, in view of 4.7, that

$$
\left\|u_{h}\right\|_{4}^{4}+\left\|v_{h}\right\|_{4}^{4}+2 b\left\|u_{h} v_{h}\right\|_{2}^{2} \rightarrow 0 .
$$


From the Ekeland variational principle we find that there exists a sequence $\left(\xi_{h}, z_{h}\right)$ such that

$$
\begin{gathered}
\left\|u_{h}-\xi_{h}\right\|_{H^{1}}+\left\|v_{h}-z_{h}\right\|_{H^{1}} \leq \sqrt{\delta}_{h}, \\
c\left(V+\eta_{h}, W+\eta_{h}\right)-\delta_{h}<I_{V+\eta_{h}, W+\eta_{h}}\left(\xi_{h}, z_{h}\right)<c\left(V+\eta_{h}, W+\eta_{h}\right)+\delta_{h}, \\
I_{V+\eta_{h}, W+\eta_{h}}^{\prime}\left(\xi_{h}, z_{h}\right) \rightarrow 0 .
\end{gathered}
$$

From 4.11 and 4.10 it follows that

$$
\left\|\xi_{h}\right\|_{4}^{4}+\left\|z_{h}\right\|_{4}^{4}+2 b\left\|\xi_{h} z_{h}\right\|_{2}^{2} \rightarrow 0 .
$$

Then

$$
\begin{aligned}
0<c^{-} & =\lim _{h \rightarrow \infty}\left\{I_{V+\eta_{h}, W+\eta_{h}}\left(\xi_{h}, z_{h}\right)-\frac{1}{2}\left\langle I_{V+\eta_{h}, W+\eta_{h}}^{\prime}\left(\xi_{h}, z_{h}\right),\left(\xi_{h}, z_{h}\right)\right\rangle\right\} \\
& =\frac{1}{4} \lim _{h \rightarrow \infty}\left\{\left\|w_{h}\right\|_{4}^{4}+\left\|z_{h}\right\|_{4}^{4}+2 b\left\|w_{h} z_{h}\right\|_{2}^{2}\right\}=0,
\end{aligned}
$$

which is an obvious contradiction, proving that $\theta\left(u_{h}, v_{h}\right)$ remains bounded. Setting $\theta(u, v)=\theta(u, v, V, W)$ and using the definition we have

$$
I_{V, W}(\theta(u, v) u, \theta(u, v) v)=\max _{t \geq 0} I_{V, W}(t u, t v) .
$$

In view of 4.3, 4.5), 4.6) and 4.8,

$$
\begin{aligned}
c(V, W) & \leq I_{V, W}\left(\theta\left(u_{h}, v_{h}\right) u_{h}, \theta\left(u_{h}, v_{h}\right) v_{h}\right) \\
& =I_{V+\eta_{h}, W+\eta_{h}}\left(\theta\left(u_{h}, v_{h}\right) u_{h}, \theta\left(u_{h}, v_{h}\right) v_{h}\right)-\frac{\eta_{h}}{2} \theta^{2}\left(u_{h}, v_{h}\right)\left(\left\|u_{h}\right\|_{2}^{2}+\left\|v_{h}\right\|_{2}^{2}\right) \\
& \leq c\left(V+\eta_{h}, W+\eta_{h}\right)+\delta_{h}-\frac{\eta_{h}}{2} \theta^{2}\left(u_{h}, v_{h}\right)\left(\left\|u_{h}\right\|_{2}^{2}+\left\|v_{h}\right\|_{2}^{2}\right) \\
& \leq c^{-}+\delta_{h}-\frac{\eta_{h}}{2} \theta^{2}\left(u_{h}, v_{h}\right)\left(\left\|u_{h}\right\|_{2}^{2}+\left\|v_{h}\right\|_{2}^{2}\right) \\
& \leq c(V, W)+\delta_{h}-\frac{\eta_{h}}{2} \theta^{2}\left(u_{h}, v_{h}\right)\left(\left\|u_{h}\right\|_{2}^{2}+\left\|v_{h}\right\|_{2}^{2}\right) .
\end{aligned}
$$

From 4.7) and as $\theta$ is a homeomorphism on the unit sphere, it follows, for $h \rightarrow \infty$, that $c(V, W)=c^{-}$. In a similar fashion one can prove that

$$
c(V, W)=\lim _{\eta \rightarrow 0^{+}} c(V+\eta, W+\eta)
$$

Therefore (4.4) is proved. Let now $\left\{z_{h}\right\}$ be a sequence in $\mathbb{R}^{3}$ such that $z_{h} \rightarrow z$ as $h \rightarrow \infty$. Observe that, given $\eta>0$, for large $h$, we have

$$
\begin{aligned}
V(z)+\eta & \geq V(z)+\left|V\left(z_{h}\right)-V(z)\right| \\
& \geq V\left(z_{h}\right) \geq V(z)-\left|V\left(z_{h}\right)-V(z)\right| \geq V(z)-\eta,
\end{aligned}
$$


and similar relations hold for $W$. From (4.4) and 4.12) we deduce that $c(V(z)+\eta$, $W(z)+\eta)$ and $c(V(z)-\eta, W(z)-\eta)$ both converge to $c(V(z), W(z))$, yielding the desired continuity of $z \mapsto \Sigma(z)$.

Let us show that the function $\Sigma$ defined in (2.9) is locally Lipschitz continuous. We denote by $\mathbb{S}(z)$ the set of nonnegative radial critical points of $I_{z}$ of least energy. Let $z, \xi \in \mathbb{R}^{3}$ and $\left(\phi_{z}, \psi_{z}\right) \in \mathbb{S}(z)$, where we write $\theta(z, \xi)=\theta\left(\phi_{z}, \psi_{z}, \xi\right)=\theta\left(\phi_{z}, \psi_{z}, V(\xi), W(\xi)\right)$. Then

$$
\Sigma(\xi)-\Sigma(z) \leq I_{\xi}\left(\theta(z, \xi)\left(\phi_{z}, \psi_{z}\right)\right)-I_{z}\left(\phi_{z}, \psi_{z}\right)
$$

Defining

$$
h(\xi)=I_{\xi}\left(\theta(z, \xi)\left(\phi_{z}, \psi_{z}\right)\right)
$$

and noting that $\theta(z, z)=1$ we obtain

$$
\Sigma(\xi)-\Sigma(z) \leq h(\xi)-h(z) .
$$

In order to prove that $\Sigma$ is locally Lipschitz, we will use the mean value theorem applied to the function $h(\xi)$, so that we will show that $\nabla h$ is bounded. First observe that since $\theta(z, \xi)\left(\phi_{z}, \psi_{z}\right) \in \mathcal{N}_{\xi}$, it follows that $\theta(z, \xi)$ is given by 4.9 with $u_{h}=\phi_{h}, v_{h}=\psi_{h}$ and $V=V(\xi), W=W(\xi)$. From the continuity of the critical level in dependence on $V(\xi), W(\xi)$ and from the continuity of $\Sigma$ we deduce that the functions

$$
\begin{aligned}
(z, \xi) & \mapsto\left\|\nabla \phi_{z}\right\|_{2}^{2}+\left\|\nabla \psi_{z}\right\|_{2}^{2}+V(\xi)\left\|\phi_{z}\right\|_{2}^{2}+W(\xi)\left\|\psi_{z}\right\|_{2}^{2}, \\
z & \mapsto\left\|\phi_{z}\right\|_{4}^{4}+\left\|\psi_{z}\right\|_{4}^{4}+2 b\left\|\phi_{z} \psi_{z}\right\|_{2}^{2}
\end{aligned}
$$

remain bounded and away from zero from below as $z$ and $\xi$ remain bounded, so that $\theta(z, \xi)$ remains bounded for $(z, \xi)$ bounded. Moreover, $\theta(z, \xi)$ is differentiable with respect to the variable $\xi$ so that also the function $h$ defined in 4.13 is differentiable and its gradient is given by

$$
\begin{aligned}
\nabla h(\xi)= & \nabla_{\xi} I_{\xi}\left(\theta(z, \xi)\left(\phi_{z}, \psi_{z}\right)\right)=\frac{\theta^{2}(z, \xi)}{2}\left[\nabla V(\xi)\left\|\phi_{z}\right\|_{2}^{2}+\nabla W(\xi)\left\|\psi_{z}\right\|_{2}^{2}\right] \\
& +\theta(z, \xi) \nabla_{\xi} \theta(z, \xi)\left[\left\|\left.\nabla \phi_{z}\right|_{2} ^{2}+\right\| \nabla \psi_{z}\left\|_{2}^{2}+V(\xi)\right\| \phi_{z}\left\|_{2}^{2}+W(\xi)\right\| \psi_{z} \|^{2}\right] \\
& -\theta^{3}(z, \xi) \nabla_{\xi} \theta(z, \xi)\left[\left\|\phi_{z}\right\|_{4}^{4}+\left\|\psi_{z}\right\|_{4}^{4}+2 b\left\|\phi_{z} \psi_{z}\right\|_{2}^{2}\right],
\end{aligned}
$$

so that

$$
\begin{aligned}
\nabla h(\xi)= & \frac{\theta^{2}(z, \xi)}{2}\left[\nabla V(\xi)\left\|\phi_{z}\right\|^{2}+\nabla W(\xi)\left\|\psi_{z}\right\|_{2}^{2}\right] \\
& +\frac{\nabla_{\xi} \theta(z, \xi)}{\theta^{2}(z, \xi)} I_{\xi}^{\prime}\left(\theta(z, \xi) \phi_{z}, \theta(z, \xi) \psi_{z}\right)\left[\theta(z, \xi) \phi_{z}, \theta(z, \xi) \psi_{z}\right] .
\end{aligned}
$$

Hence, since $\left(\theta(z, \xi) \phi_{z}, \theta(z, \xi) \psi_{z}\right) \in \mathcal{N}_{\xi}$, we get

$$
\nabla h(\xi)=\frac{\theta^{2}(z, \xi)}{2}\left[\nabla V(\xi)\left\|\phi_{z}\right\|^{2}+\nabla W(\xi)\left\|\psi_{z}\right\|_{2}^{2}\right]
$$


This formula, (4.14), the mean value theorem applied to the function $h$ and the local boundedness of $\theta$ imply that $\Sigma$ is locally Lipschitz (in order to get the opposite inequality, it suffices to switch $z$ with $\xi$ ).

Now, let us prove conclusion (a) of Theorem 2.8 Let $z \in \mathcal{E}$ and $\left(u_{\varepsilon_{n}}, v_{\varepsilon_{n}}\right) \subset \mathbb{H}$ a sequence of solutions to $\left(S_{\varepsilon}\right)$ that satisfy the properties in Definition 2.6 For all $n \geq 1$ consider $\varepsilon_{n} \rightarrow 0$ and the sequences $\varphi_{n}(x)=u_{\varepsilon_{n}}\left(z+\varepsilon_{n} x\right), \psi_{n}(x)=v_{\varepsilon_{n}}\left(z+\varepsilon_{n} x\right)$, so that $\varphi_{n}(x)+\psi_{n}(x) \rightarrow 0$ as $|x| \rightarrow \infty$, uniformly with respect to $n$, and $J_{\varepsilon_{n}}\left(\varphi_{n}, \psi_{n}\right) \rightarrow \Sigma(z)$ as $n \rightarrow \infty$. The sequence $\left(\varphi_{n}, \psi_{n}\right) C^{2}$ converges over compact sets to a least energy solution $\left(\varphi_{z}, \psi_{z}\right)$ of $\left[S_{z}\right]$, and $\varphi_{z}, \psi_{z}$ are radially and exponentially decaying (see [10]), that is, $\left(\varphi_{z}, \psi_{z}\right)$ belongs to $\mathbb{S}(z)$.

Consider the scalar problems

$$
\begin{aligned}
& \left\{\begin{array}{l}
-\Delta u+V(z) u=u^{3} \quad \text { in } \mathbb{R}^{3}, \\
u>0, \quad u \in H^{1}, \\
u(0)=\max _{\mathbb{R}^{3}} u,
\end{array}\right. \\
& \left\{\begin{array}{l}
-\Delta v+W(z) v=v^{3} \quad \text { in } \mathbb{R}^{3}, \\
v>0, \quad v \in H^{1}, \\
v(0)=\max _{\mathbb{R}^{3}} v .
\end{array}\right.
\end{aligned}
$$

It is known (see [8], [16]) that $\left[S_{z}^{V}\right]$ and $\left(S_{z}^{W}\right)$ have a unique ground state solution. Notice that Proposition 3.3 implies that, if $z \in \mathcal{O}_{b}$, then $\left(\varphi_{z}, \psi_{z}\right)$ has necessarily one trivial component. So that, the following possibilities may occur:

I. $z \in \mathcal{O}_{b}$ and $\varphi_{z}=0$ and $\psi_{z}$ is a nontrivial solution to $S_{z}^{W}$;

II. $z \in \mathcal{O}_{b}$ and $\psi_{z}=0$ and $\varphi_{z}$ is a nontrivial solution to $\left(S_{z}^{V}\right)$;

III. $z \in \mathcal{E} \backslash \mathcal{O}_{b}=\mathcal{E}_{\Sigma}$.

It is readily seen by a simple scaling that, if $\varphi_{z} \neq 0$ or $\psi_{z} \neq 0$,

$$
\varphi_{z}(x)=\sqrt{V(z)} U_{0}(\sqrt{V(z)} x), \quad \psi_{z}(x)=\sqrt{W(z)} U_{0}(\sqrt{W(z)} x),
$$

where $U_{0}$ is the unique solution to $-\Delta u+u=u^{3}$. Since $\psi_{n}$ converges uniformly to $\psi_{z}$, which has its global maximum point at the origin, case I corresponds to $z \in \mathcal{E}_{W}$. In such a case, in light of (4.1), we have $\gamma_{1}(z)=0, \gamma_{2}(z) \neq 0$, that is, $z \in \operatorname{Crit}(W)$. Arguing as above it is possible to show that the situation of case II implies that $z \in \mathcal{E}_{V}$ and $z \in \operatorname{Crit}(V)$. Of course $\mathcal{E}_{V} \cap \mathcal{E}_{W} \cap\{V \neq W\}=\emptyset$. Indeed, if $z^{*} \in \mathcal{E}_{V} \cap \mathcal{E}_{W} \cap\{V \neq$ $W\}$ there would exist two sequences $\left(u_{j}^{1}, v_{j}^{1}\right)$ and $\left(u_{j}^{2}, v_{j}^{2}\right)$ of solutions to $\left.S_{\varepsilon}\right)$ such that the corresponding scaled solutions $\left(\varphi_{j}^{1}, \psi_{j}^{1}\right)$ and $\left(\varphi_{j}^{2}, \psi_{j}^{2}\right)$ converge in the $C^{2}$ sense over compact sets to $\left(\varphi_{z^{*}}^{1}, \psi_{z^{*}}^{1}\right) \in \mathbb{S}\left(z^{*}\right)$ and $\left(\varphi_{z^{*}}^{2}, \psi_{z^{*}}^{2}\right) \in \mathbb{S}\left(z^{*}\right)$ respectively, and $\varphi_{j}^{1}(0) \geq \delta>$ 0 (since $z^{*} \in \mathcal{E}_{V}$ ) and $\psi_{j}^{2}(0) \geq \delta>0$ (since $z^{*} \in \mathcal{E}_{W}$ ), for every $j$. As a consequence, letting $j \rightarrow \infty$, we get $\varphi_{z^{*}}^{1} \neq 0$ and $\psi_{z^{*}}^{2} \neq 0$. Now, in light of Proposition 3.3. since 
$z^{*} \in \mathcal{O}_{b}$ and $\left(\varphi_{z^{*}}^{1}, \psi_{z^{*}}^{1}\right)$ and $\left(\varphi_{z^{*}}^{2}, \psi_{z^{*}}^{2}\right)$ have least energy, we have $\psi_{z^{*}}^{1}=0$ and $\varphi_{z^{*}}^{2}=0$. Therefore,

$$
\Gamma \sqrt{V\left(z^{*}\right)}=I_{z^{*}}\left(\varphi_{z^{*}}^{1}, 0\right)=\Sigma\left(z^{*}\right)=I_{z^{*}}\left(0, \psi_{z^{*}}^{2}\right)=\Gamma \sqrt{W\left(z^{*}\right)},
$$

contrary to $V\left(z^{*}\right) \neq W\left(z^{*}\right)$. The previous facts show that

$$
\mathcal{E} \cap \mathcal{O}_{b} \subseteq \mathcal{E}_{V} \cup \mathcal{E}_{W} \quad \text { and } \quad \mathcal{E}_{V} \times \mathcal{E}_{W} \subset \operatorname{Crit}(V) \times \operatorname{Crit}(W) .
$$

Hence, we conclude that

$$
\mathcal{E}=\left(\mathcal{E} \cap \mathcal{O}_{b}\right) \cup\left(\mathcal{E} \backslash \mathcal{O}_{b}\right)=\mathcal{E}_{V} \cup \mathcal{E}_{W} \cup \mathcal{E}_{\Sigma},
$$

with $\mathcal{E}_{V} \times \mathcal{E}_{W} \subset \operatorname{Crit}(V) \times \operatorname{Crit}(W)$. To prove conclusion (a) of Theorem 2.8 it is only left to show that $\mathcal{E}_{\Sigma} \subset \mathrm{Crit}_{C}(\Sigma)$. In order to do this we will first prove that the directional derivatives from the left and right of $\Sigma$ at every point $z \in \mathbb{R}^{3}$ along any $\eta \in \mathbb{R}^{3}$ exist, and

$$
\left(\frac{\partial \Sigma}{\partial \eta}\right)^{-}(z)=\sup _{\left(\varphi_{z}, \psi_{z}\right) \in \mathbb{S}(z)} \frac{\partial I_{z}}{\partial \eta}\left(\varphi_{z}, \psi_{z}\right), \quad\left(\frac{\partial \Sigma}{\partial \eta}\right)^{+}(z)=\inf _{\left(\varphi_{z}, \psi_{z}\right) \in \mathbb{S}(z)} \frac{\partial I_{z}}{\partial \eta}\left(\varphi_{z}, \psi_{z}\right),
$$

that is, explicitly,

$$
\begin{aligned}
& \left(\frac{\partial \Sigma}{\partial \eta}\right)^{-}(z)=\sup _{\left(\varphi_{z}, \psi_{z}\right) \in \mathbb{S}(z)} \frac{1}{2}\left\{\frac{\partial V}{\partial \eta}(z)\left\|\varphi_{z}\right\|_{2}^{2}+\frac{\partial W}{\partial \eta}(z)\left\|\psi_{z}\right\|_{2}^{2}\right\}, \\
& \left(\frac{\partial \Sigma}{\partial \eta}\right)^{+}(z)=\inf _{\left(\varphi_{z}, \psi_{z}\right) \in \mathbb{S}(z)} \frac{1}{2}\left\{\frac{\partial V}{\partial \eta}(z)\left\|\varphi_{z}\right\|_{2}^{2}+\frac{\partial W}{\partial \eta}(z)\left\|\psi_{z}\right\|_{2}^{2}\right\},
\end{aligned}
$$

for every $z, \eta \in \mathbb{R}^{3}$.

Let $\left\{\mu_{j}\right\} \subset \mathbb{R}^{3}$ be a sequence converging to $\mu_{0}$ and let $\left(u_{j}, v_{j}\right)$ be a corresponding sequence of solutions of least energy $\Sigma\left(\mu_{j}\right)$. We want to prove that, up to a subsequence, $u_{j} \rightarrow u_{0}$ and $v_{j} \rightarrow v_{0}$ strongly in $H^{1}$, with $\left(u_{0}, v_{0}\right) \in \mathbb{S}\left(\mu_{0}\right)$. It is straightforward to see that $\left(u_{j}, v_{j}\right)$ is bounded in $H^{1} \times H^{1}$ so that, up to a subsequence, it converges weakly to a pair $\left(u_{0}, v_{0}\right)$, and $u_{j} \rightarrow u_{0}$ and $v_{j} \rightarrow v_{0}$ locally in the $C^{2}$ sense, so that $\left(u_{0}, v_{0}\right)$ is a solution to the limiting problem with $\mu=\mu_{0}$. Moreover, as previously observed, there exists $\delta>0$ such that $u_{0}^{2}(0)+v_{0}^{2}(0) \geq \delta$, which entails $u_{0} \neq 0$ or $v_{0} \neq 0$. Observe that, by the continuity of $\Sigma$ and by Fatou's lemma, we get

$$
\Sigma\left(\mu_{0}\right)=\lim _{j \rightarrow \infty} \Sigma\left(\mu_{j}\right)=\lim _{j \rightarrow \infty} I_{\mu_{j}}\left(u_{j}, v_{j}\right) \geq I_{\mu_{0}}\left(u_{0}, v_{0}\right) \geq \Sigma\left(\mu_{0}\right) .
$$

Hence, in particular, $I_{\mu_{j}}\left(u_{j}, v_{j}\right) \rightarrow I_{\mu_{0}}\left(u_{0}, v_{0}\right)=\Sigma\left(\mu_{0}\right)$ as $j \rightarrow \infty$, that is,

$$
\begin{aligned}
\lim _{j \rightarrow \infty} \int_{\mathbb{R}^{3}}\left[\left|\nabla u_{j}\right|^{2}+\left|\nabla v_{j}\right|^{2}+V\left(\mu_{j}\right) u_{j}^{2}+W\left(\mu_{j}\right) v_{j}^{2}\right] \\
=\int_{\mathbb{R}^{3}}\left[\left|\nabla u_{0}\right|^{2}+\left|\nabla v_{0}\right|^{2}+V\left(\mu_{0}\right) u_{0}^{2}+W\left(\mu_{0}\right) v_{0}^{2}\right] .
\end{aligned}
$$


Thus $\left(u_{j}, v_{j}\right) \rightarrow\left(u_{0}, v_{0}\right)$ strongly in $H^{1} \times H^{1}$. For any $(\varphi, \psi) \in \mathbb{S}(z)$, we get

$$
\begin{aligned}
\Sigma(z+t \eta)-\Sigma(z) & \leq I_{z+t \eta}(\vartheta(z, z+t \eta) \varphi, \vartheta(z, z+t \eta) \psi)-I_{z}(\varphi, \psi) \\
& =\left.t \nabla_{\xi} I_{\xi}(\vartheta(\xi, z) \varphi, \vartheta(\xi, z) \psi)\right|_{\xi \in[z, z+t \eta]} .
\end{aligned}
$$

Hence, by the arbitrariness of $(\varphi, \psi) \in \mathbb{S}(z)$,

$$
\limsup _{t \rightarrow 0^{+}} \frac{\Sigma(z+t \eta)-\Sigma(z)}{t} \leq \inf _{(\varphi, \psi) \in \mathbb{S}(z)} \frac{1}{2}\left\{\nabla V(z) \cdot \eta\|\varphi\|_{2}^{2}+\nabla W(z) \cdot \eta\|\psi\|_{2}^{2}\right\} .
$$

To get the opposite inequality, take $(\varphi, \psi) \in \mathbb{S}(z+t \eta)$. Then

$$
\begin{aligned}
\Sigma(z+t \eta)-\Sigma(z) & \geq I_{z+t \eta}(\varphi, \psi)-I_{z}(\theta(z+t \eta, z) \varphi, \theta(z+t \eta, z) \psi) \\
& =\left.t \nabla_{\xi} I_{\xi}(\theta(\xi, z+t \eta) \varphi, \theta(\xi, z+t \eta) \psi)\right|_{\xi \in[z, z+t \eta]}
\end{aligned}
$$

Using the continuity of $\theta$ and the convergence of $(\varphi, \psi)$ to an element of $\mathbb{S}(z)$, we obtain

$$
\liminf _{t \rightarrow 0^{+}} \frac{\Sigma(z+t \eta)-\Sigma(z)}{t} \geq \inf _{(\varphi, \psi) \in \mathbb{S}(z)} \frac{1}{2}\left\{\nabla V(z) \cdot \eta\|\varphi\|_{2}^{2}+\nabla W(z) \cdot \eta\|\psi\|_{2}^{2}\right\},
$$

proving the opposite inequality, so that the desired formula for the right derivative of $\Sigma$ follows. A similar argument provides the corresponding formula for the left derivative.

Assume now that $z \in \mathcal{E} \backslash \mathcal{O}_{b}=\mathcal{E}_{\Sigma}$. Notice that, by [4.2], for all $\eta \in \mathbb{R}^{3}$,

$$
\int_{\mathbb{R}^{3}}\left[\frac{\partial V}{\partial \eta}(z) \varphi_{z}^{2}+\frac{\partial W}{\partial \eta}(z) \psi_{z}^{2}\right]=0 .
$$

Hence, since $\left(\varphi_{z}, \psi_{z}\right) \in \mathbb{S}(z)$, by formula (4.15) we have

$$
\left(\frac{\partial \Sigma}{\partial \eta}\right)^{+}(z) \leq 0
$$

Then, by the definition of $(-\Sigma)^{0}(z ; \eta)$, we get

$$
(-\Sigma)^{0}(z ; \eta) \geq\left(\frac{\partial(-\Sigma)}{\partial \eta}\right)^{+}(z) \geq 0 \quad \text { for every } \eta \in \mathbb{R}^{3} .
$$

In turn $0 \in \partial_{C}(-\Sigma)(z)$ and, since $\partial_{C}(-\Sigma)(z)=-\partial_{C} \Sigma(z)$ (see [11]), we obtain $z \in$ $\mathrm{Crit}_{C}(\Sigma)$, which concludes the proof of (a).

If $V$ and $W$ are also bounded from above, by choosing $b_{0}^{\infty}$ and $b_{1}^{\infty}$ as in 2.14-2.15 we get $\mathcal{O}_{b}=\mathbb{R}^{3}$ for all $b \leq b_{0}^{\infty}$ (as $b_{0}^{\infty} \leq b_{z}$ for every $z$ ), and $\mathcal{O}_{b}=\emptyset$ for all $b>b_{1}^{\infty}$ (as $b_{1}^{\infty} \geq b_{z}$ for every $z$ ), thus immediately proving assertion (b). Finally, if $b>b_{2}^{\infty}$ the last assertion of the theorem follows immediately from Proposition 3.3

Acknowledgments. The first and the second authors are supported by the MIUR national research project "Variational Methods and Nonlinear Differential Equations", while the third author is supported by the MIUR national research project "Variational and Topological Methods in the Study of Nonlinear Phenomena" and by the Istituto Nazionale di Alta Matematica (INdAM). 


\section{References}

[1] Ambrosetti, A., Badiale, M., Cingolani, S.: Semiclassical states of nonlinear Schrödinger equation. Arch. Ration. Mech. Anal. 140, 285-300 (1997) Zbl 0896.35042 MR 1486895

[2] Ambrosetti, A., Colorado, E.: Bound and ground states of coupled nonlinear Schrödinger equations. C. R. Math. Acad. Sci. Paris 342, 453-458 (2006) Zbl pre05018096 MR 2214594

[3] Ambrosetti, A., Colorado, E.: Standing waves of some coupled nonlinear Schrödinger equations. J. London Math. Soc. 75, 67-82 (2007)

[4] Ambrosetti, A., Malchiodi, A.: Perturbation Methods and Semilinear Elliptic Problems on $\mathbb{R}^{n}$. Progr. Math. 240, Birkhäuser, Basel (2006) Zbl pre05013399 MR 2186962

[5] Ambrosetti, A., Malchiodi, A., Secchi, S.: Multiplicity results for some nonlinear Schrödinger equations with potentials. Arch. Ration. Mech. Anal. 159, 253-271 (2001) Zbl 1040.35107 MR 1857674

[6] Ambrosetti, A., Rabinowitz, P. H.: Dual variational methods in critical points theory and applications. J. Funct. Anal. 14, 349-381 (1973) Zbl 0273.49063 MR 0370183

[7] Bartsch, T., Wang, Z. Q.: Note on ground states of nonlinear Schrödinger systems. J. Partial Differential Equations 19, 200-207 (2006) Zbl 1104.35048 MR 2252973

[8] Berestycki, H., Lions, P.-L.: Nonlinear scalar fields equation I. Existence of a ground state. Arch. Ration. Mech. Anal. 82, 313-346 (1983) Zbl 0533.35029 MR 0695535

[9] Brezis, H., Lieb, E. H.: Minimum action solutions of some vector field equations. Comm. Math. Phys. 96, 97-113 (1984) Zbl 0579.35025 MR 0765961

[10] Busca, J., Sirakov, B.: Symmetry results for semi-linear elliptic systems in the whole space. J. Differential Equations 163 (2000), 41-56. Zbl 0952.35033 MR 1755067

[11] Clarke, F. H.: Optimization and Nonsmooth Analysis. Wiley-Interscience Publ. (1983) Zbl 0582.49001 MR 0709590

[12] Coffman, C. V.: Uniqueness of the ground state solution for $\Delta u-u+u^{3}=0$ and a variational characterization of other solutions. Arch. Ration. Mech. Anal. 46, 81-95 (1972) Zbl 0249.35029 MR 0333489

[13] Del Pino, M., Felmer, P.: Local mountain passes for semi-linear elliptic problems in unbounded domains. Calc. Var. Partial Differential Equations 4, 121-137 (1996) Zbl 0844.35032 MR 1379196

[14] Fibich, G., Papanicolau, G.: Self-focusing in the perturbed and unperturbed nonlinear Schrödinger equation in critical dimension. SIAM J. Appl. Math. 60, 183-240 (1999) Zbl 1026.78013 MR 1740841

[15] Floer, A., Weinstein, A.: Nonspreading wave packets for the cubic Schrödinger equation with a bounded potential. J. Funct. Anal. 69, 397-408 (1986) Zbl 0613.35076 MR 0867665

[16] Kwong, M. K.: Uniqueness of positive solutions of $\Delta u-u+u^{p}=0$ in $\mathbb{R}^{N}$. Arch. Ration. Mech. Anal. 105, 243-266 (1989) Zbl 0676.35032 MR 0969899

[17] Lin, T. C., Wei, J.: Ground state of $N$ coupled nonlinear Schrödinger equations in $\mathbb{R}^{n}, n \leq 3$. Comm. Math. Phys. 255, 629-653 (2005) Zbl pre02214972 MR 2135447

[18] Maia, L. A., Montefusco, E., Pellacci, B.: Positive solutions for a weakly coupled nonlinear Schrödinger system. J. Differential Equations 229, 743-767 (2006) Zbl 1104.35053 MR 2263573

[19] Menyuk, C. R.: Nonlinear pulse propagation in birefringent optical fibers. IEEE J. Quantum Electron. 23, 174-176 (1987)

[20] Oh, Y. G.: Existence of semiclassical bound state of nonlinear Schrödinger equations. Comm. Math. Phys. 209, 223-243 (1993) 
[21] Pomponio, A.: Coupled nonlinear Schrödinger systems with potentials. J. Differential Equations 227, 258-281 (2006) Zbl 1100.35098 MR 2233961

[22] Pucci, P., Serrin, J.: A general variational identity. Indiana Univ. Math. J. 35, 681-703 (1986) Zbl 0625.35027 MR 0855181

[23] Rabinowitz, P. H.: On a class of nonlinear Schrödinger equations. Z. Angew. Math. Phys. 43, 270-291 (1992) Zbl 0763.35087 MR 1162728

[24] Secchi, S., Squassina, M.: On the location of concentration points for singularly perturbed elliptic equations. Adv. Differential Equations 9, 221-239 (2004) Zbl pre05054520 MR 2099612

[25] Sirakov, B.: Least energy solitary waves for a system of nonlinear Schrödinger equations in $\mathbb{R}^{n}$. Comm. Math. Phys. 271, 199-221 (2007) MR 2283958

[26] Strauss, W. A.: Existence of solitary waves in higher dimensions. Comm. Math. Phys. 55, 149-162 (1977) Zbl 0356.35028 MR 0454365

[27] Wang, X., Zeng, B.: On concentration of positive bound states of nonlinear Schrödinger equations with competing potential functions. SIAM J. Math. Anal. 28, 633-655 (1997) Zbl 0879.35053 MR 1443612 